\title{
Transmit Power Minimization for Wireless Networks with Energy Harvesting Relays
}

\author{
Yaming Luo, Jun Zhang, Senior Member, IEEE, and Khaled B. Letaief, Fellow, IEEE
}

\begin{abstract}
Energy harvesting (EH) has recently emerged as a key technology for green communications as it can power wireless networks with renewable energy sources. However, directly replacing the conventional non-EH transmitters by EH nodes will be a challenge. In this paper, we propose to deploy extra EH nodes as relays over an existing non-EH network. Specifically, the considered non-EH network consists of multiple source-destination (S-D) pairs. The deployed EH relays will take turns to assist each S-D pair, and energy diversity can be achieved to combat the low $\mathrm{EH}$ rate of each $\mathrm{EH}$ relay. To make the best of these EH relays, with the source transmit power minimization as the design objective, we formulate a joint power assignment and relay selection problem, which, however, is NPhard. We thus propose a general framework to develop efficient sub-optimal algorithms, which is mainly based on a sufficient condition for the feasibility of the optimization problem. This condition yields useful design insights and also reveals an energy hardening effect, which provides the possibility to exempt the requirement of non-causal EH information. Simulation results will show that the proposed cooperation strategy can achieve near-optimal performance and provide significant power savings. Compared to the greedy cooperation method that only optimizes the performance of the current transmission block, the proposed strategy can achieve the same performance with much fewer relays, and the performance gap increases with the number of S-D pairs.
\end{abstract}

Index Terms-Energy harvesting communications, power assignment, relay selection, energy diversity, cooperative communications.

\section{INTRODUCTION}

Wireless communications is evolving towards a green paradigm with significantly improved energy efficiency and environmental friendliness [1]. In particular, energy harvesting $(\mathrm{EH})$ technology has recently emerged as a promising approach to power wireless networks with renewable energy sources [2, 3]. The EH node can obtain energy from the environment [4], including solar energy, thermoelectric energy, vibration energy, RF energy, etc. As the harvested energy is clean and renewable, environmental friendliness is innate in EH networks. Besides this, by exempting the manual battery replacement, EH technology also qualifies the wireless networks with the ability of virtually perpetual operation [5, 6]. These advantages render EH technology a good candidate for green communications.

This work is supported by the Hong Kong Research Grant Council under Grant No. 610212.

The authors are with the Dept. of Electronic and Computer Engineering, Hong Kong University of Science and Technology, Hong Kong. Email: \{luoymhk, eejzhang, eekhaled\}@ ust.hk. Khaled B. Letaief is also with Hamad Bin Khalifa University, Qatar (kletaief@hbku.edu.qa).
A clean-slate design of a wireless network solely powered by energy harvesting will, however, be challenging, if not impossible. Typically the harvested energy is in a small amount [7] and is also spatially-temporally varying [8]. Hence, it will be difficult to provide satisfactory communication performance in an EH network. Meanwhile, communications protocols need to be re-designed to handle the random and intermittent energy arrivals. On the other hand, considering the implementation cost and efficiency, the existing communication infrastructure should be fully utilized. Therefore, it is more realistic to incrementally incorporate the energy harvesting feature into existing non-EH wireless networks. In this paper, we will propose to deploy multiple EH relays to assist the transmission of multiple communication pairs in an existing network, so that the conventional power consumption of the source nodes can be significantly reduced. For fairness consideration, in this paper, we consider the maximum power consumption minimization problem, similar to the minimum rate maximization problem for throughput-oriented optimization with fairness consideration [9]. If the transmitters are mobile devices powered by batteries, this kind of optimization is directly related to the extension of the network lifetime, which is of particular importance for many applications such as wireless sensor networks [10]. The optimization of network operations in such a network will require new cooperation strategies, which form the main focus of this paper.

\section{A. Related Works}

The potential of EH technology has recently spurred a lot of research activities in the wireless communications community. There are two main design approaches, i.e. offline and online policies, depending on non-causal and causal side information (of the energy state or the channel state), respectively. The offline transmission policies were investigated in [11-15] for different kinds of networks, ranging from the point-to-point channel, broadcast channel, multiple access channel, to interference channel. The online algorithms, mainly for the pointto-point channel, were designed in [16-19]. These studies have revealed the main challenges in designing EH networks, which are caused by the energy causality constraint, i.e. the energy consumed so far cannot exceed the total harvested energy. Subsequently, communication protocols need to be revisited in EH networks. For example, even when the channel remains unchanged, the transmit power should still adapt to the random energy arrivals [17]. Moreover, the communication performance is fundamentally limited by the small-amount and time-varying energy arrivals. 
Cooperative communications has been proven to be an effective technique to improve the communication performance as well as the energy efficiency for wireless networks [20,-24]. In particular, its potential in $\mathrm{EH}$ wireless networks has been recently investigated. For single-relay two-hop EH networks, the authors in [25. 31] investigated the problem of power allocation and source/relay transmission scheduling, and verified that the existing transmission policies for conventional nonEH communication systems perform poorly. With multiple $\mathrm{EH}$ relays, it turns out that the cooperation strategy design is very challenging due to the EH constraints. In [32], the SER performance of a multi-relay EH network using a simple relay selection scheme based on the instantaneous side information of each relay was analyzed thoroughly. In particular, the coupling effect among different relays was observed and discussed. Joint power allocation and relay selection was considered in [33] with either non-causal or causal channel and energy side information, but the complexity of the proposed algorithms is high. By adopting a fixed transmit power, an efficient but sub-optimal relay selection method based on the so-called relative throughput gain was proposed in [34].

All the relay selection methods in [32-[34] require the channel side information of each relay in each transmission block, which is challenging to obtain, especially given the low $\mathrm{EH}$ rates of the EH relays. Previous works on EH networks focused on the cumulative performance over a relatively long duration, while the short-term performance during a particular transmission block may not satisfy the application requirement. This short-term performance is normally a special design obstacle we need to overcome for $\mathrm{EH}$ systems, due to the time-varying $\mathrm{EH}$ rate at each $\mathrm{EH}$ node. Moreover, only a single source-destination (S-D) pair has been considered in the previous studies of EH relaying networks. With multiple S-D pairs, besides the dynamic energy arrivals at each EH relay, the spatial distribution of all the EH relays and S-D pairs will sharpen the design challenges for cooperation strategies.

In this paper, we aim to achieve power conservation for non-EH wireless networks composed of multiple S-D pairs by deploying multiple EH relays. For this system, the cooperation strategies in previous works are no longer applicable. To be practical, we assume that each source node does not have instantaneous channel state information. To guarantee satisfactory performance of all the S-D pairs, for each transmission block each source needs to transmit with the assistance of relays. Meanwhile, a certain QoS requirement is used to regulate the communication performance. Note that such a network with multiple S-D pairs is very generic in practice [9], especially for ad-hoc networks and mesh networks.

\section{B. Contributions}

In this paper, we propose an effective and low-complexity cooperation strategy to reduce the transmit power of multiple S-D pairs with the help of EH relays. To combat the low EH rate of the relays, energy diversity will be achieved by allowing relays to take turns to forward the source information. The main contributions are summarized as follows.

- To optimize the proposed cooperation strategy, we will formulate a joint power assignment and relay selection problem. The design objective is to minimize the maximum transmit power among all the S-D pairs. This problem is found to be NP-hard, and we will propose a general framework to develop efficient sub-optimal algorithms. The key step is to derive an easy-to-check sufficient condition for the feasibility of the optimization problem. The proposed algorithms are of low complexity, and the optimality can be achieved when the number of transmission blocks is sufficiently large.

- With the sufficient condition, the roles of key system parameters as well as design insights for such EH relay networks will be obtained. Similar to the channel hardening effect in massive MIMO systems, a kind of energy hardening effect in such multi-relay EH networks is observed. We find that when the number of EH relays is large, there will be little performance loss if only using the instantaneous energy side information, without the offline energy side information.

- We shall demonstrate via simulations that the proposed cooperation strategy can achieve significant power savings over the direct link transmission without EH relays. We will also show that the proposed strategy outperforms the greedy strategy that only optimizes the current block transmission. Specifically, the proposed strategy requires much fewer relays to achieve the same performance, and the performance gap between the two strategies becomes larger when the number of S-D pairs increases. Moreover, the proposed sub-optimal algorithms provide performance close to the corresponding performance upper bound, and they have the potential to be extended to other cooperative EH networks.

The rest of this paper is organized as follows. We shall describe the system model and problem formulation in Section II. We will first solve the respective feasibility problem with a single S-D pair in Section III. The complete solution for the original optimization problem is provided in Section IV. In Section V, simulation results are then presented to demonstrate the advantage of the proposed design approach. Finally, Section VI concludes the paper.

Notation: Matrices are denoted by bold-face upper-case letters. Sets are denoted by calligraphic upper-case letters such as $\mathcal{A}$, and $|\mathcal{A}|$ represents its cardinality. 0 denotes an all-zero matrix. $x \leftarrow y$ represents assigning the value of $y$ to $x$. For two sets $\mathcal{A}$ and $\mathcal{B}, \mathcal{A}-\mathcal{B}$ is the relative complement of $\mathcal{A}$ with respect to $\mathcal{B} . \mathbf{1}_{\varphi}$ denotes the indicator function. $\varphi_{1} \Leftrightarrow \varphi_{2}$ means that $\varphi_{1}$ is sufficient and necessary for $\varphi_{2}$. $\lceil x\rceil(\lfloor x\rfloor)$ means the ceiling (floor) function.

\section{System Model AND Problem Formulation}

The main design objective of this paper is to reduce the power consumption in an existing wireless network with $M$ source-destination (S-D) pairs. The proposed approach is to deploy $K \mathrm{EH}$ relays to forward information for these S-D pairs. Consequently, the considered network becomes a relay-assisted multi-source multi-destination network, as shown in Fig. 11. The set of S-D pair indices is denoted as 
$\mathcal{M}=\{1,2, \ldots, M\}$, and the set of relay indices is denoted as $\mathcal{K}=\{1,2, \ldots, K\}$. In the following part of this section, we will first describe the details of the system model, and present the proposed cooperation strategy. To optimize the cooperation strategy, we will then formulate the design problem to be tackled in this paper.

\section{A. System Setting}

We assume that all the channels are block fading, with the coherence time denoted as $T^{\mathrm{C}}$, corresponding to one transmission block. In the first half of one transmission block, each source transmits its information signal, while in the second half, the selected relay forwards the information to the corresponding destination. We consider the system design within a given transmission duration of length $T$, which consists of $N=\frac{T}{T^{\mathrm{C}}}$ transmission blocks, with $N$ normally much larger than $K$. Denote the set of all the transmission block indices in $T$ as $\mathcal{N}=\{1,2, \ldots, N\}$. To guarantee satisfactory performance of all the S-D pairs, we assume that for each transmission block, each source needs to transmit with the assistance of relays. Meanwhile, a certain QoS requirement is used to regulate the communication performance. To be practical, we assume that the source nodes do not have instantaneous channel state information as the EH relays may not have enough energy for channel training/feedback. Therefore, the design will be based on the statistical channel information and the EH rate of each relay.

Each source is assumed to use a fixed transmit power, denoted as $P_{\mathrm{s}, m}^{\mathrm{tr}}$ for the $m$-th source, but the value of $P_{\mathrm{s}, m}^{\mathrm{tr}}$ needs to be properly determined. Denote the transmit power vector of all sources as $\mathbf{P}_{\mathrm{s}}=\left[P_{\mathrm{s}, m}^{\mathrm{tr}}\right]$. In this paper, we assume that different S-D pairs are coordinated to transmit within different frequency bands, and thus there will be no cochannel interference [9, 21]. Even for such an interference-free case, the design problem turns out to be challenging, and the obtained results will be helpful for investigating more general cases with interference. All the relays are half-duplex and apply the amplify-and-forward (AF) relaying protocol, while the extension to other relaying protocols is straightforward. There is a per band peak transmit power constraint for each relay, denoted as $P_{k, \text { max }}^{\mathrm{tr}}$ for the $k$-th relay for each frequency band it uses. For reference, the main notations are listed in Table I.

\section{B. Energy Harvesting Model}

An important factor that determines the performance of an EH communication node is the EH profile, which models the cumulative harvested energy up to time $t$. For the $k$-th $\mathrm{EH}$ relay, denote the $\mathrm{EH}$ profile as $E_{k, \Sigma}^{\mathrm{EH}}(t)$, and the initial energy in the battery as $E_{k}^{\text {init }}=E_{k, \Sigma}^{\mathrm{EH}}(0)$. We will consider a piecewise constant model for the EH profile. That is, each EH node has a steady but low EH rate, and the EH rate for the $k$-th relay in the $n$-th transmission block is denoted as $P_{k, n}^{\mathrm{EH}}$. The change of the $\mathrm{EH}$ rate is in the time unit of an $\mathrm{EH}$ interval $T^{\mathrm{E}}$. As the $\mathrm{EH}$ rate usually does not vary frequently, $T^{\mathrm{E}}$ is normally much larger than the channel coherence time $T^{\mathrm{C}}$. A similar EH model is adopted in [18, 34], and it can be used for such energy

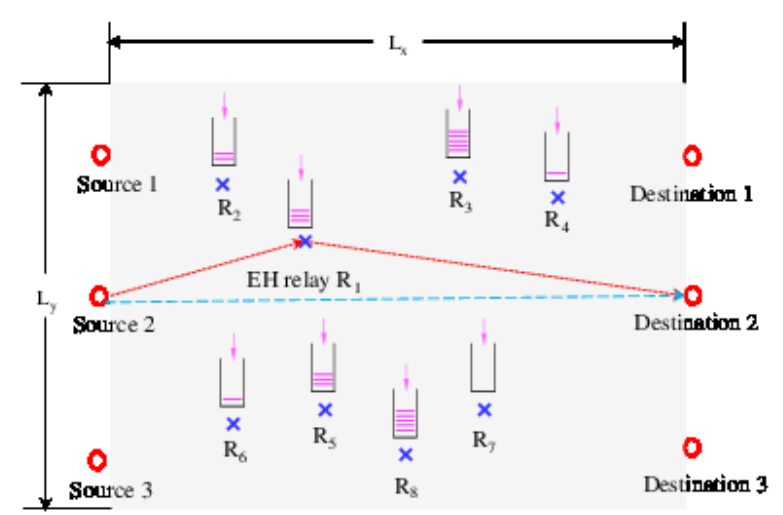

Figure 1: An example system with 3 S-D pairs, where the sources are non-EH nodes. To reduce the transmit power of this network, we deploy 8 EH relays.

Table I: Main notations

\begin{tabular}{|c||l|}
\hline Symbols & Definition \\
\hline \hline $\mathcal{M}$ & S-D pair set with cardinality $M$ \\
\hline $\mathcal{K}$ & Relay set with cardinality $K$ \\
\hline $\mathcal{N}$ & Transmission block set with cardinality $N$ \\
\hline$T^{\mathrm{C}}$ & Transmission block length \\
\hline$T^{\mathrm{E}}$ & Energy harvesting interval \\
\hline$T$ & Total transmission duration \\
\hline$N$ & Number of blocks in $T$ \\
\hline$E_{k, \Sigma}^{\mathrm{EH}}(t)$ & EH profile at time $t$ of the $k$-th relay \\
\hline$E_{k}^{\text {init }}$ & Initial energy of the $k$-th relay \\
\hline$P_{k, n}^{\mathrm{EH}}$ & EH rate of $k$-th relay in $n$-th transmission block \\
\hline$E_{k,,}(t)$ & Cumulative energy at time $t$ for the $k$-th relay \\
\hline$P_{k, \text { max }}^{\mathrm{tr}}$ & Maximum transmit power of the $k$-th relay \\
\hline
\end{tabular}

sources as solar energy [35]. We assume $T^{\mathrm{E}}=N^{\mathrm{C}} T^{\mathrm{C}}$, i.e. the $j$-th EH interval includes the transmission blocks $(j-1) T^{\mathrm{C}}+$ 1 to $j T^{\mathrm{C}}$, where $j=1,2, \ldots, N^{\mathrm{E}}$. Moreover, $T=N^{\mathrm{E}} T^{\mathrm{E}}$, and $N=N^{\mathrm{E}} N^{\mathrm{C}}$. For simplicity, we denote the set of all the EH interval indices as $\mathcal{J}=\left\{1,2, \ldots, N^{\mathrm{E}}\right\}$. As the $\mathrm{EH}$ rate, which we should denote by $\Psi_{k, j}$, stays constant in the $j$-th EH interval, then

$$
P_{k, n_{1}}^{\mathrm{EH}}=P_{k, n_{2}}^{\mathrm{EH}}=\Psi_{k, j},
$$

$\forall n_{1}, n_{2}$, such that $\left\lceil\frac{n_{1}}{N^{\mathrm{C}}}\right\rceil=\left\lceil\frac{n_{2}}{N^{\mathrm{C}}}\right\rceil=j$. Moreover, we assume that the EH process for each relay is a stationary random process. The EH rates for different relays or among different $\mathrm{EH}$ intervals, i.e. $P_{k, n}^{\mathrm{EH}}$ with different $k$ or $n$, are independent of each other. Similar to [12, 17], we assume that offline EH information of all EH relays is available, which is valid for some predictable energy resources, such as solar energy. With such information, we can obtain a performance upper bound for the case with causal energy information, and also reveal some properties that provide design guidelines for more general cases. In Section III.B, we find that when the number of EH relays is large enough, the dependence on the future energy information is negligible, which provides the possibility of only using the current energy side information in the multirelay EH networks. The full solution for general EH networks with causal energy information will be left to our future work. 
For an EH node, the utilization of the harvested energy is constrained by the EH profile, which yields the energy causality constraint [28]. The energy causality means that the energy consumed thus far cannot exceed the total harvested energy. For the $k$-th EH relay, denote the instantaneous power consumption as $P_{k}(t)$, and then the energy causality constraint can be expressed as

$$
\int_{0}^{t} P_{k}(\tau) d \tau \leq E_{k, \Sigma}^{\mathrm{EH}}(t)
$$

This constraint will bring major design challenges for the EH networks. In particular, for the system in Fig. 11 it will cause a coupling effect when determining the transmit powers of different relays and selecting different relays, as will be discussed in the following sub-section.

In this paper, we only consider the energy consumption for information transmission, while ignoring other types of energy consumption. For each EH relay, we assume that the battery capacity is large enough, while the more general case is left for future work. Moreover, we assume that the initial energy in the battery for the $k$-th relay can support its maximum transmit power in all the frequency bands it supports, i.e. $E_{k}^{\text {init }}=M P_{k, \max }^{\operatorname{tr}} T^{\mathrm{C}} / 2$. This is mainly to guarantee the performance during the first few transmission blocks.

\section{Proposed Cooperation Strategy}

In the proposed cooperation strategy, different relays will take turns to assist the transmissions of all the S-D pairs. The main components of the proposed cooperation strategy include power assignment and relay selection, which are jointly determined.

1) Power Assignment: As our transmit power optimization design is based on statistical channel information, we do not consider the power adaptation of each node over time. Once the transmit power is determined, the value will be fixed within the whole transmission duration, and the relay will use the same transmit power whenever it is selected to forward information. Power assignment determines the transmit power for each relay. For a given relay, when assisting a certain S-D pair, a low transmit power cannot provide a good performance. Whereas, a too high transmit power will exhaust its energy too soon, implying that some other relays will be selected more often. Besides this, there also exists a coupling effect among different S-D pairs, as each relay possibly serves multiple S-D pairs with only limited energy. The coupling effect complicates the system design, and thus the power assignment needs to be carefully decided. We denote the transmit power matrix of all relays as $\mathbf{P}_{\mathrm{r}}=\left[P_{m, k}^{\mathrm{tr}}\right]$, where $P_{m, k}^{\mathrm{tr}}$ is the transmit power of the $k$-th relay when assisting the $m$-th S-D pair.

2) Relay Selection: For the proposed cooperation strategy, relay selection will be performed in each transmission block, and it should be based on each relay's available energy. A relay is called active if its available energy, denoted as $E_{k, \Sigma}(t)$, is enough to support its assigned transmit power, i.e.

$$
E_{k, \Sigma}(t) \geq P_{m, k}^{\mathrm{tr}} T^{\mathrm{C}} / 2 .
$$

In each transmission block, one active relay will be selected to assist each S-D pair. Denote the relay selection matrix as $\mathrm{Z}=\left[z_{m, k, n}\right]$ where

$$
z_{m, k, n}=\left\{\begin{array}{ll}
1 & m \text {-th pair selects } k \text {-th relay in } n \text {-th block } \\
0 & \text { otherwise }
\end{array} .\right.
$$

Given $\mathrm{Z}$, the available energy for the $k$-th relay before the relay transmission stage of the $l$-th transmission block can be expressed as

$$
\begin{gathered}
E_{k, \Sigma}\left(\left(l-\frac{1}{2}\right) T^{\mathrm{C}}\right)=E_{k}^{\mathrm{init}}+\sum_{n=1}^{l-1} P_{k, n}^{\mathrm{EH}} T^{\mathrm{C}} \\
+P_{k, l}^{\mathrm{EH}} \frac{1}{2} T^{\mathrm{C}}-\sum_{m=1}^{M} \sum_{n=1}^{l-1} z_{m, k, n} P_{m, k}^{\mathrm{tr}} \frac{1}{2} T^{\mathrm{C}}
\end{gathered}
$$

Since $\sum_{k=1}^{K} z_{m, k, n} \leq 1, n \in \mathcal{N}, m \in \mathcal{M}$, we can at most select one relay for each pair in each transmission block, and thus the decision of whether to select one relay for a certain transmission block is coupled with that of other relays. For each feasible relay selection matrix $\mathrm{Z}$, different relays would take turns to forward the source information.

Remark 1. With the proposed cooperation strategy, different relays will take turns to assist the S-D communications. Consequently, each of them will have time to accumulate enough energy for transmission, despite the limited harvested energy in each single transmission block. This strategy bears a similar motivation as diversity in wireless communications [36]. With diversity, multiple copies of the same information will be sent through links with independent fading. Hence, it is very unlikely that all the links will be weak. Therefore, a so-called diversity gain is achieved. On the other hand, for our proposed cooperation strategy, the hope is that we can always find an EH relay with enough energy to help the S$\mathrm{D}$ communication, and the achieved performance gain can be regarded as the energy diversity gain [37].

\section{Problem Formulation}

To guarantee satisfactory performance of all the S-D pairs, we assume that there is a QoS constraint for each pair, and it is measured by a general utility function [38], which is a monotonically increasing function of the allocated resource. The utility may represent the successful transmission probability, transmission rate, or other QoS metric, depending on the application scenario. For the $m$-th source with transmit power $P_{\mathrm{s}, m}^{\mathrm{tr}}$, when the $k$-th relay assists it with transmit power $P_{m, k}^{\mathrm{tr}}$, the achieved utility is denoted as $U_{m, k}\left(P_{\mathrm{s}, m}^{\mathrm{tr}}, P_{m, k}^{\mathrm{tr}}\right)$. Based on the relay selection matrix Z, for the $m$-th S-D pair in the $n$-th transmission block, with $m \in \mathcal{M}, n \in \mathcal{N}$, we have $U^{(m, n)}=\sum_{k=1}^{K} z_{m, k, n} U_{m, k}\left(P_{\mathrm{s}, m}^{\mathrm{tr}}, P_{m, k}^{\mathrm{tr}}\right)$. In order to guarantee steady and reliable communications, we assume that the QoS constraint shall be satisfied in each transmission block for each S-D pair. Thus, the QoS constraint is expressed as $U^{(m, n)} \geq U_{\text {th }}$. Upon this, $\sum_{k=1}^{K} z_{m, k, n}=1$ shall hold for all $n \in \mathcal{N}, m \in \mathcal{M}$, i.e. each pair will select one relay in each 
transmission block. Otherwise, the QoS requirement will be violated.

The design objective is to minimize the maximum transmit power among all the S-D pairs, i.e. to attain power conservation for all the sources at the same time. The design problem can then be formulated as

\section{OP1:}

$$
\begin{aligned}
\min _{\mathbf{P}_{\mathrm{s}}, \mathbf{P}_{\mathrm{r}}, \mathbf{z}} & \max _{m} P_{m, s}^{\mathrm{tr}} \\
\text { s.t. } & \sum_{m=1}^{M} \sum_{n=1}^{l} z_{m, k, n} P_{m, k}^{\mathrm{tr}} \frac{1}{2} T^{\mathrm{C}} \leq E_{k}^{\mathrm{init}}+\sum_{n=1}^{l-1} P_{k, n}^{\mathrm{EH}} T^{\mathrm{C}} \\
& +P_{k, l}^{\mathrm{EH}} \frac{1}{2} T^{\mathrm{C}}, \forall l \in \mathcal{N}, k \in \mathcal{K} \\
& P_{m, k}^{\mathrm{tr}} \leq P_{k, \mathrm{max}}^{\mathrm{tr}}, \forall k \in \mathcal{K}, m \in \mathcal{M} \\
& U^{(m, n)} \geq U_{\mathrm{th}}, \forall n \in \mathcal{N}, m \in \mathcal{M} \\
& \sum_{k=1}^{K} z_{m, k, n}=1, \forall n \in \mathcal{N}, m \in \mathcal{M} \\
& z_{m, k, n} \in\{0,1\}, \forall m \in \mathcal{M}, k \in \mathcal{K}, n \in \mathcal{N}
\end{aligned}
$$

where (5) is based on the energy causality constraint (2), (6) is the peak power constraint for each relay, (7) is the QoS constraint, and (8) guarantees that for each transmission block, one relay is selected for each S-D pair.

OP1 is a joint power assignment and relay selection optimization problem, i.e. we jointly design $\mathbf{P}$ and $\mathbf{Z}$. It is a highly complicated problem, as it belongs to the mixedinteger nonlinear programming (MINLP) problem [39], which is known to be NP-hard. Moreover, one particular difficulty is the coupling effect among the operations for different relays, and another comes from the large variable size. The worstcase complexity is exponential with the source number $M$, the relay number $K$, and the transmission block number $N$. We will consider the following equivalent epigraph form of OP1

$$
\begin{aligned}
\text { OP2: } & \\
\min _{\mathbf{P}_{\mathrm{s}}, \mathbf{P}_{\mathrm{r}}, \mathbf{Z}, \eta} & \eta \\
\text { s.t. } & P_{m, s}^{\mathrm{tr}} \leq \eta, \forall m \in \mathcal{M}, \\
& \text { Constraints } \sim 5] .97 .
\end{aligned}
$$

In the following, we will first investigate the feasibility problem for OP2 with a given $\eta$,

$$
\begin{aligned}
\text { FP1: } & \\
\text { find } & \mathbf{P}_{\mathrm{s}}, \mathbf{P}_{\mathrm{r}}, \mathbf{Z}, \\
\text { s.t. } & P_{m, s}^{\mathrm{tr}} \leq \eta, \forall m \in \mathcal{M}, \\
& \text { Constraints } 5 \text { (5), }
\end{aligned}
$$

which will then help develop a general design framework and efficient algorithms for the original problem. Particularly, it can be checked that the feasibility of FP1 with various $\eta$ has a two-phase pattern. If FP1 is feasible for a given $\eta$, it must be feasible for any $\eta^{\prime}>\eta$. On the other hand, if it is infeasible for a given $\eta$, then it must be infeasible for any $\eta^{\prime}<\eta$. Hence, we can deduce that the smallest $\eta$ that can make FP1 feasible corresponds to the optimal solution of the original optimization problem OP1. Therefore, the major task now lies in solving the feasibility problem FP1 with a given $\eta$. In the following, we will start by investigating FP1 for the single-pair case, i.e. $M=1$, in section III. The solution is then generalized to solve the feasibility problem FP1 with multiple pairs. Based on this feasibility study, the solution to the original problem OP1 will be obtained. Details of the proposed algorithm for OP1 will be provided in section IV.

\section{A Special Case: Feasibility Study for a Single S-D PAIR}

In this section, we will deal with the feasibility problem FP1 with a single S-D pair and a given $\eta$. For the notations in this section, the pair index $m$ will be removed. Particularly, the relay transmit power assignment matrix $\mathbf{P}_{\mathrm{r}}$ is reduced to a vector, and the source transmit power vector to a scalar.

With a single S-D pair, the feasibility problem FP1 with a given $\eta$ is

$$
\begin{array}{cl}
\text { FP2: } & \\
\text { find } & P_{s}^{\mathrm{tr}}, \mathbf{P}_{\mathrm{r}}, \mathbf{Z} \\
\text { s.t. } & P_{s}^{\mathrm{tr}} \leq \eta, \\
& \sum_{n=1}^{l} z_{k, n} P_{k}^{\mathrm{tr}} \frac{1}{2} T^{\mathrm{C}} \leq E_{k}^{\mathrm{init}}+\sum_{n=1}^{l-1} P_{k, n}^{\mathrm{EH}} T^{\mathrm{C}} \\
& +P_{k, l}^{\mathrm{EH}} \frac{1}{2} T^{\mathrm{C}}, \forall l \in \mathcal{N}, k \in \mathcal{K}, \\
& P_{k}^{\mathrm{tr}} \leq P_{k, \mathrm{max}}^{\mathrm{tr}}, \forall k \in \mathcal{K}, \\
& U^{(n)} \geq U_{\mathrm{th}}, \forall n \in \mathcal{N}, \\
& \sum_{k}^{K} z_{k, n}=1, \forall n \in \mathcal{N}, \\
& z_{k=1} \in\{0,1\}, \forall k \in \mathcal{K}, n \in \mathcal{N} .
\end{array}
$$

In the following, we will first reformulate this problem into a simpler form, which is still NP-hard, but can help derive a sufficient condition for its feasibility. The scenarios where the sufficient condition becomes necessary will also be tackled.

\section{A. Problem Reformulation}

In this sub-section, we will remove constraints 10 and (13) by introducing the operation of relay pre-selection and fixing the relay transmit power $\mathbf{P}_{\mathrm{r}}$, respectively. Meanwhile, the variable size will be greatly reduced.

With a given $\eta$, for the $k$-th relay, if $U_{k}\left(\eta, P_{k, \max }^{\mathrm{tr}}\right)<U_{\text {th }}$, then this relay should not be selected even once, i.e. $z_{k, n}=0$, $\forall n \in \mathcal{N}$. Otherwise, constraint 13 will be violated. On the other hand, if $U_{k}\left(\eta, P_{k, \max }^{\mathrm{tr}}\right) \geq U_{\mathrm{th}}$, it will be selected at least once within the transmission duration $T$, i.e. $\sum_{n=1}^{N} z_{k, n} \geq 1$, given that $N \geq K$. Hence, without loss of generality, we can just let the source select these relays one by one in the first several transmission blocks. This can be done until all these relays have been selected once, without affecting the feasibility.

Based on the above insights, we define the set of such relays as the candidate relay subset. The procedure of determining 
the candidate relay subset is called relay pre-selection. Therefore, the temporary ${ }^{1}$ relay pre-selection result with a given $\eta$ is determined as

$$
\mathcal{S}_{\eta}=\left\{k \in \mathcal{K} \mid U_{k}\left(\eta, P_{k, \max }^{\mathrm{tr}}\right) \geq U_{\text {th }}\right\} .
$$

For notational simplicity and with a given $\eta$ and an arbitrary $x$, denote $g_{k, \eta}(x)=U_{k}(\eta, x)$, and the inverse function of $g_{k, \eta}(x)$ over $x$ as $g_{k, \eta}^{-1}(\cdot)$. For $k \in \mathcal{S}_{\eta}$, the minimum transmit power of the $k$-th relay that can meet the QoS constraint $U_{\text {th }}$ is determined as $g_{k, \eta}^{-1}\left(U_{\text {th }}\right)$. For a given $\eta$, this minimum transmit power only depends on the relay location. We denote $\hat{\mathbf{P}}_{\eta}=$ $\left[\hat{P}_{k}(\eta)\right]$ where

$$
\hat{P}_{k}(\eta)=\left\{\begin{array}{ll}
g_{k, \eta}^{-1}\left(U_{\text {th }}\right) & k \in \mathcal{S}_{\eta} \\
0 & k \in \mathcal{K}-\mathcal{S}_{\eta}
\end{array} .\right.
$$

Then, we have the following property that can simplify the feasibility checking of FP2:

Lemma 1. FP2 is feasible $\Leftrightarrow \boldsymbol{F P 2}$ with $\mathbf{P}_{\mathrm{r}}=\hat{\mathbf{P}}_{\eta}$ is feasible.

Proof: We prove the equivalence in two steps. We will first show the necessity, and then the sufficiency. The necessity is obvious, as by fixing the transmit power, the feasible domain shrinks. If the shrunken domain is still feasible, so is the original one. For the sufficiency part, and based on the definition of $P_{k}^{*}(\eta)$ in $16, P_{k}^{*}(\eta)$ is the smallest power value satisfying $(10)$. With any given $\mathbf{Z}$, if $P_{k}^{*}(\eta)$ violates $(11)$ or (12), no other power value can satisfy (11) and (12). Therefore, if the feasible domain with fixed $P_{k}^{\mathrm{tr}}=P_{k}^{*}(\eta)$ is empty, so is the feasible domain with other power values.

With Lemma 1, we can replace the power vector $\mathbf{P}_{\mathrm{r}}$ in FP2 by $\hat{\mathbf{P}}_{\eta}$ and also remove Eq. 13 . Therefore, when checking the feasibility of FP2, we do not need to check the full domain of the power vector $\mathbf{P}_{\mathrm{r}}$, but need only to check a single vector $\hat{\mathbf{P}}_{\eta}$, which largely reduces the complexity. Moreover, with a given $\mathcal{S}_{\eta}$, and based on the expression of the utility function $U^{(m, n)}$ in the last section, we only need to have $U_{m, k}\left(\eta, P_{k, \text { max }}^{\mathrm{tr}}\right) \geq$ $U_{\text {th }}$, and $\sum_{k \in \mathcal{S}_{\eta}} z_{k, n}=1, \forall n \in \mathcal{N}$, which means that there are active relays for each transmission block and all of these relays can satisfy the QoS constraint. Therefore, (10) can also be removed from $\mathbf{F P 2}$.

Based on the above discussion, we have fixed $\mathbf{P}_{\mathrm{r}}$ and removed constraints $(10)$ and $(13)$, and thus we obtain the following equivalent problem for FP2:

FP3:

$$
\begin{array}{ll}
\text { find } & \mathbf{Z} \\
\text { s.t. } & \sum_{n=1}^{l} \frac{1}{2} \hat{P}_{k}(\eta) T^{\mathrm{C}} z_{k, n} \leq E_{k}^{\mathrm{init}}+\sum_{n=1}^{l-1} P_{k, n}^{\mathrm{EH}} T^{\mathrm{C}} \\
& +P_{k, l}^{\mathrm{EH}} \frac{1}{2} T^{\mathrm{C}}, \forall l \in \mathcal{N}, k \in \mathcal{S}_{\eta}, \\
& \sum_{k \in \mathcal{S}_{\eta}} z_{k, n}=1, \forall n \in \mathcal{N}, z_{k, n} \in\{0,1\}, \\
& \forall k \in \mathcal{S}_{\eta}, n \in \mathcal{N} .
\end{array}
$$

\footnotetext{
${ }^{1}$ This relay pre-selection result $\mathcal{S}_{\eta}$ is only a "temporary" result, as it is for a given $\eta$, while in general it will be different from the final result.
}

Compared with FP2, the dimension of FP3 is reduced as $\mathbf{P}_{r}$ is fixed, and some constraints are removed. It can be verified that FP3 is the respective feasibility problem of a multi-resource generalized assignment problem (MRGAP) [40], which is still NP-hard. A particular difficulty is the large size of $\mathbf{Z}$. Therefore, although the problem has been significantly simplified, it is still difficult to deal with. In the following, by exploiting the relationship of several key system parameters, we will derive a sufficient condition for the feasibility of FP3, which is easy to check. This will then be used to provide a sub-optimal algorithm for the original optimization problem OP2.

\section{B. Condition for Feasibility}

In this part, despite the complexity of the problem, we will propose a simple condition to check the feasibility of the problem, which is sufficient, and will become necessary for some asymptotic scenarios.

Define the cumulative average $\mathrm{EH}$ rate up to the end of the $j$-th EH interval for the $k$-th relay as $\bar{\Psi}_{k, j}=\frac{\sum_{i=1}^{j} \Psi_{k, j}}{j}$, $\forall j \in \mathcal{J}$. With the given $\eta$ and $l$, denote

$$
\zeta(\eta, l)=\underbrace{\sum_{k \in \mathcal{S}_{\eta}} \frac{2 \bar{\Psi}_{k, l}}{\hat{P}_{k}(\eta)}}_{\zeta_{1}(\eta, l)}+\underbrace{\sum_{k \in \mathcal{S}_{\eta}} \frac{2 E_{k}^{\mathrm{init}} / T^{\mathrm{C}}-\hat{P}_{k}(\eta)}{\hat{P}_{k}(\eta) l N^{\mathrm{C}}}}_{\zeta_{2}(\eta, l)} .
$$

We briefly introduce the insights from the expression of $\zeta(\eta, l)$ in Eq. [19). The two parts of $\zeta(\eta, l)$ play different roles in the system operation. The first term, $\zeta_{1}(\eta, l)$, can be regarded as the steady-state component, which manipulates the long-term ${ }^{2}$ balance of the harvested energy and consumed energy. On the other hand, the second term, $\zeta_{2}(\eta, l)$, is specifically designed to improve the performance at the beginning stage of the whole transmission duration. The value of $\zeta_{2}(\eta, l)$ is computed based on the approximation that all relays' harvested energy and initial energy are exactly used up in the end. Meanwhile the initial energy for each relay is enough for the transmit energy of one packet. With or without the second term $\zeta_{2}(\eta, l)$, the sufficiency always holds. But the performance without this term will be poor in the case where the transmission block number is not too large. Based on Eq. (19) we have the following result, which provides a simple sufficient condition to check the feasibility of FP3.

Theorem 1. The following is a sufficient condition for the feasibility of $\boldsymbol{F P 3}$ :

$$
\min _{j \in \mathcal{J}} \zeta(\eta, j) \geq 1
$$

Particularly, for two asymptotic scenarios, with other parameters fixed, we have the following properties:

1. The necessity property: When $N^{\mathrm{C}} \rightarrow \infty$, the sufficient condition is necessary.

\footnotetext{
${ }^{2}$ Here "long term" refers to the part that still remains after an extremely large number of transmission blocks.
} 
2. The deterministic property: When $K \rightarrow \infty, \zeta(\eta, l)$ becomes deterministic ${ }^{3}$ independent of the concrete realization of $\left[P_{k}^{\mathrm{EH}}\right]$.

Proof: The proof is given in the Appendix.

As shown in the proof, once condition (20) is satisfied, there exist active relays for each transmission block. In this case, different relays will take turns to assist the S-D pair and the unsatisfactory performance caused by the low EH rate at a single relay can be overcome. Moreover, based on Theorem 1. and given that the effect of the large-sized matrix $\mathbf{Z}$ has been removed, condition 20 only depends on $\hat{\mathbf{P}}_{\eta}$, and thus it is easy to check.

Next, we will discuss the insights that can be obtained from Theorem 1:

1. Insights from the condition (20) on the whole. Based on the expression of $\zeta(\eta, l)$ for each given $l$, the minimization of $\zeta(\eta, l)$ over $l$ in condition 20 is to adapt to different $\mathrm{EH}$ rates over different EH intervals. For condition $[20$, we can find that increasing $\eta$ has two effects $-\hat{P}_{k}(\eta)$ will increase according to $(16)$, while the set $\mathcal{S}_{\eta}$ may shrink. Therefore, a larger $\eta$ makes it easier to violate condition $(20)$. That is, the achievable performance for $\mathbf{O P 2}$ will be limited. In order to improve it, it is better to have more relays with higher average EH rates and lower $\hat{P}_{k}(\eta)$ (corresponding to a more favorable location).

2. Insights from the necessity property. When the number of transmission blocks is large, the condition becomes sufficient and necessary, and the performance loss caused by using this condition instead of directly solving FP3 disappears. Moreover, the later simulation results will show that the convergence speed is very fast. In particular, to approach the close-to-optimal performance, only tens of transmission blocks are enough, which can be naturally satisfied by the practical system parameters.

3. Insights from the deterministic property. When the relay number is large, besides the energy diversity mentioned in the last section, another phenomenon occurs. That is, the randomness from each single EH relay vanishes gradually. Similar to the channel hardening effect in massive MIMO systems [41], this kind of effect can be called the energy hardening effect. The offline $\mathrm{EH}$ information, which is very important for the optimal design in EH networks [11], is usually not easy to obtain in practical systems, especially when the number of EH nodes is large. Fortunately, the energy hardening effect makes it possible that the performance loss due to the lack of offline EH information becomes extremely small, as long as the relay number is large. This will avoid the necessity of predicting future $\mathrm{EH}$ rates for systems with a large number of relays, and thus will greatly simplify the protocol designs.

\footnotetext{
${ }^{3}$ Here we only consider the non-trivial cases. For some $\eta$, it is possible that $\zeta(\eta, j)$ becomes infinity. But in this case, the condition is already satisfied trivially, and the problem FP3 can be easily verified to be feasible. This corresponds to the case where the system is with enough harvested energy from these relays. In this case, the value of $\eta$ can be further reduced given the relays' EH situations, and this $\eta$ cannot be optimal for the original OP2 Here we ignore such trivial cases.
}

\section{Proposed Algorithm for Transmit Power MINIMIZATION}

In this section, we will first investigate the feasibility problem with multiple S-D pairs, with the help of the results for the single-pair case. We will then propose an efficient bisection algorithm to solve the original joint power assignment and relay selection problem.

\section{A. The Feasibility Problem with Multiple S-D pairs}

In this subsection, we will investigate the feasibility problem with multiple S-D pairs. Compared with the single S-D pair case, a special difficulty lies in the coupling effect among different S-D pairs as each relay is expected to assist multiple pairs during the whole transmission duration. At first, we will divide the problem into several sub-problems by introducing new variables. This procedure can be physically understood as relay division. Next, for each sub-problem, we exploit the results for the single-pair case to simplify it. Finally, jointly considering all the sub-problems together, the complexity is further squeezed. Note that, among the previously-mentioned steps, all the other steps preserve the equivalence except the one applying the single-pair case result, which contributes only sub-optimality.

1) Problem Decoupling: In this part, we will reformulate the problem into a decoupled version by introducing new variables, and this procedure will be later physically interpreted as a procedure of relay division.

We are faced with a relay set in which an arbitrary relay is possible to serve an arbitrary S-D pair, and each relay needs to determine its power allocation for different pairs. Therefore, for a certain relay, the decision of how to assist one S-D pair is coupled with the decision for another pair. Such a coupling effect greatly complicates the problem. Note that the coupling effect among different S-D pairs is caused by constraint (5). Fortunately, by introducing two new variable sets $\left[\phi_{m, k}\right]$ and $\left[\theta_{m, k, n}\right]$, it can be verified that the feasibility of (5) is equivalent to that of the following decoupled one.

$$
\begin{aligned}
& \sum_{n=1}^{l} z_{m, k, n} P_{m, k}^{\mathrm{tr}} \frac{T^{\mathrm{C}}}{2} \leq \phi_{m, k} E_{k}^{\mathrm{init}}+\sum_{n=1}^{l-1} \theta_{m, k, n} P_{k, n}^{\mathrm{EH}} T^{\mathrm{C}} \\
& \quad+\theta_{m, k, l} P_{k, l}^{\mathrm{EH}} \frac{T^{\mathrm{C}}}{2}, \forall l, k, m, \\
& \quad 0 \leq \phi_{m, k} \leq 1,0 \leq \theta_{m, k, n} \leq 1, \forall m \in \mathcal{M}, k \in \mathcal{K}, n \in \mathcal{N}, \\
& \quad \sum_{m=1}^{M} \phi_{m, k}=1, \sum_{m=1}^{M} \theta_{m, k, n}=1, \forall k \in \mathcal{K}, n \in \mathcal{N} .
\end{aligned}
$$

Remark 2. The previous transformation from (5) to 21) has a clear physical meaning as relay division. We consider the energy flow through a certain relay. Based on Eq. (5), for the $k$-th relay, the initial energy is $E_{k}^{\text {init. }}$. In the $n$-th transmission block, the entering energy is from the EH process with $\mathrm{EH}$ rate $P_{k, n}^{\mathrm{EH}}$, while the exiting part includes the transmit powers for all the S-D pairs it possibly serves, i.e. $\sum_{m=1}^{M} z_{m, k, n} P_{m, k}^{\mathrm{tr}}$. While for Eq. 21, with given values of $\left[\phi_{m, k}\right]$ and $\left[\theta_{m, k, n}\right]$, we can equivalently interpret 21] as an individual relay only serving the $m$-th S-D pair, and is denoted as the $(m, k)$-th child relay, with initial energy as $\phi_{m, k} E_{k}^{\text {init }}$, harvested power as $\theta_{m, k, n} P_{k, n}^{\mathrm{EH}}$, and transmit power as $P_{m, k}^{\mathrm{tr}}$. To avoid any 
confusion, the original relay is called the parent relay. In this case, through $\left[\phi_{m, k}\right]$ and $\left[\theta_{m, k, n}\right]$, the original relay set $\mathcal{K}$ is equivalently transformed into a new set including only the $M K$ child relays.

Based on the previous transformation, with given $\left[\phi_{m, k}\right]$ and $\left[\theta_{m, k, n}\right]$, the original feasibility problem $\mathbf{F P 1}$ can be divided into $M$ sub-problems, and the $m$-th $(m \in \mathcal{M})$ sub-problem is

\section{FP4:}

$$
\begin{aligned}
\text { find } & P_{m, s}^{\mathrm{tr}}, P_{m, k}^{\mathrm{tr}}, z_{m, k, n}, \forall k \in \mathcal{K}, n \in \mathcal{N} \\
\text { s.t. } & P_{m, s}^{\mathrm{tr}} \leq \eta \\
& \sum_{n=1}^{l} z_{m, k, n} P_{m, k}^{\mathrm{tr}} \frac{T^{\mathrm{C}}}{2} \leq \phi_{m, k} E_{k}^{\mathrm{init}}+\sum_{n=1}^{l-1} \theta_{m, k, n} P_{k, n}^{\mathrm{EH}} T^{\mathrm{C}} \\
& +\theta_{m, k, l} P_{k, l}^{\mathrm{EH}} \frac{T^{\mathrm{C}}}{2}, \forall l \in \mathcal{N}, k \in \mathcal{K}, \\
& P_{m, k}^{\mathrm{tr}} \leq P_{k, \max }^{\mathrm{tr}}, U_{m, k}\left(P_{\mathrm{s}, m}^{\mathrm{tr}}, P_{m, k}^{\mathrm{tr}}\right) \geq U_{\mathrm{th}}, \forall k \in \mathcal{K}, \\
& \sum_{k=1}^{K} z_{m, k, n}=1, \forall n \in \mathcal{N}, \\
& z_{m, k, n} \in\{0,1\}, \forall k \in \mathcal{K}, n \in \mathcal{N} .
\end{aligned}
$$

2) Exploiting the Solution for Single S-D Pair: For the $m$ th sub-problem in FP4, only a single S-D pair is involved, and we can use the results of Theorem 1. Similarly, we have the temporary candidate relay subset for the $m$-th S-D pair:

$$
\mathcal{S}_{\eta, m}=\left\{k \in \mathcal{K} \mid U_{m, k}\left(\eta, P_{k, \text { max }}^{\mathrm{tr}}\right) \geq U_{\mathrm{th}}\right\},
$$

and the cumulative average $\mathrm{EH}$ rate of the $(m, k)$-th child relay up to the $j$-th $\mathrm{EH}$ interval as

$$
\bar{P}_{m, k, j}^{\mathrm{EH}}=\frac{\sum_{i=1}^{j N^{\mathrm{C}}} \theta_{m, k, i} P_{k, i}^{\mathrm{EH}}}{j N^{\mathrm{C}}} .
$$

Moreover, with a given $\eta$ and an arbitrary $x$, denote $g_{m, k, \eta}(x)=U_{m, k}(\eta, x)$, and the inverse function $g_{m, k, \eta}(x)$ over $x$ as $g_{m, k, \eta}^{-1}(\cdot)$. For $k \in \mathcal{S}_{\eta, m}$, the minimum transmit power of the $k$-th relay that can meet the QoS requirement $U_{\text {th }}$ for the $m$-th S-D pair is determined as $g_{m, k, \eta}^{-1}\left(U_{\text {th }}\right)$. We denote $\hat{\mathbf{P}}_{\eta}=\left[\hat{P}_{m, k}(\eta)\right]$ where

$$
\hat{P}_{m, k}(\eta)=\left\{\begin{array}{ll}
g_{m, k, \eta}^{-1}\left(U_{\text {th }}\right) & k \in \mathcal{S}_{\eta, m} \\
0 & k \in \mathcal{K}-\mathcal{S}_{\eta, m}
\end{array} .\right.
$$

With the given $\eta$ and $l$, denote $\zeta_{m}(\eta, l)=$ $\sum_{k \in \mathcal{S}_{\eta, m}} \frac{2 \bar{P}_{m, k, l} l N^{\mathrm{C}}+2 \phi_{m, k} E_{k}^{\text {init }} / T^{\mathrm{C}}-2 \hat{P}_{k}(\eta)}{\hat{P}_{m, k}(\eta) l N^{\mathrm{C}}}$. Referring to Theorem 11 we can verify that the feasibility of the following problem is necessary for that of FP4, jointly considering all the $M$ sub-problems in FP4:

FP5: find $\phi_{m, k}, \theta_{m, k, n}, \forall k \in \mathcal{K}, n \in \mathcal{N}, m \in \mathcal{M}$

$$
\begin{array}{ll}
\text { s.t. } & \sum_{k \in \mathcal{S}_{\eta, m}} \frac{2 \bar{P}_{m, k, j}^{\mathrm{EH}} j N^{\mathrm{C}}+2 \phi_{m, k} E_{k}^{\mathrm{init}} / T^{\mathrm{C}}-2 \hat{P}_{k}(\eta)}{j N^{\mathrm{C}} \hat{P}_{m, k}(\eta)} \geq 1, \\
& \forall j \in \mathcal{J}, m \in \mathcal{M}, \\
& 0 \leq \phi_{m, k} \leq 1,0 \leq \theta_{m, k, n} \leq 1, \forall m \in \mathcal{M} \\
& k \in \mathcal{K}, n \in \mathcal{N} \\
& \sum_{m=1}^{M} \phi_{m, k}=1, \sum_{m=1}^{M} \theta_{m, k, n}=1, \\
& \forall k \in \mathcal{K}, n \in \mathcal{N} .
\end{array}
$$

Complexity is greatly squeezed from FP1 to FP5, as the complexity for FP5 is linear with $\sqrt{N} M K$. However, FP5 still suffers from the large size of those new variables $\left[\phi_{m, k}\right]$ and $\left[\theta_{m, k, n}\right]$. Next we will try to further reduce this complexity by investigating the special properties of the problem.

3) Complexity Reduction: Note that $\left[\theta_{m, k, n}\right]$ is a threedimensional matrix, and the size of the third dimension $n$ is possible to be extremely large in practice. Whereas, we find that its size can be greatly reduced with the piece-wise constant property of the EH profile. The main result is listed in the following lemma.

Lemma 2. The feasibility of FP5 is uninfluenced by setting constant values for $\theta_{m, k, n}$ within one EH interval, i.e. setting $\theta_{m, k, n_{1}}=\theta_{m, k, n_{2}}=\theta_{m, k}^{j}, \forall n_{1}, n_{2}$, such that $\left\lceil\frac{n_{1}}{N^{c}}\right\rceil=$ $\left\lceil\frac{n_{2}}{N^{\mathrm{C}}}\right\rceil=j, 1 \leq j \leq N^{\mathrm{E}}$. Thus, we can replace 23. with the following expression:

$$
\bar{P}_{m, k, j}^{\mathrm{EH}}=\frac{\sum_{i=1}^{j} \theta_{m, k}^{i} \Psi_{k, i}}{j} .
$$

Proof: Because $\forall n_{1}, n_{2}$, such that $\left\lceil\frac{n_{1}}{\Delta C}\right]=\left\lceil\frac{n_{2}}{N C}\right]=j$, $P_{k, n_{1}}^{\mathrm{EH}}=P_{k, n_{2}}^{\mathrm{EH}}=\Psi_{k, j}$. Thus, for both 23] and 20p only the sum of such $\theta_{m, k, n}$ within each EH interval matters, i.e. $\sum_{n=l N^{\mathrm{C}}+1}^{(l+1) N^{\mathrm{C}}} \theta_{m, k, n}$ for each $l$ such that $1 \leq l \leq N^{\mathrm{E}}$.

Using Lemma 2, matrix $\left[\theta_{m, k, n}\right]$ with size $M \times K \times N$ is greatly reduced to $\left[\theta_{m, k}^{j}\right]$ with size $M \times K \times N^{\mathrm{E}}$. However, the complexity is still high. In particular, the feasibility of Eq. 28 for a certain $j=j^{*} \leq N^{\mathrm{E}}$ is largely influenced by all the values of $\theta_{m, k}^{j}$ for $1 \leq j \leq j^{*}$. That is, the decisions of $\left[\theta_{m, k}^{j}\right]$ for different $\mathrm{EH}$ intervals (i.e. different $j$ ) are coupled with each other. To resolve this issue, we will propose a method to decouple $\left[\theta_{m, k}^{j}\right]$ at different EH intervals. The main results are listed in the following lemma.

Lemma 3. The feasibility of FP5 is uninfluenced by separating all $\theta_{m, k}^{j}$ from the procedure of calculating a cumulative average in Eq. (28), i.e. the feasibility remains unchanged by transforming (28) into the following expression:

$$
\bar{P}_{m, k, j}^{\mathrm{EH}}=\frac{\sum_{i=1}^{j} \Psi_{k, i}}{j} \tilde{\theta}_{m, k}^{j},
$$

where $\tilde{\theta}_{m, k}^{j}=\frac{\sum_{i=1}^{j} \Psi_{k, i} \theta_{m, k}^{i}}{\sum_{i=1}^{j} \Psi_{k, i}}$. Moreover, the new variable $\left[\tilde{\theta}_{m, k}^{j}\right]$ also satisfies the same constraints for $\left[\theta_{m, k}^{j}\right]$ in 26 and 27 . 
Proof: The equivalence can be verified by utilizing the relationship between $\left[\theta_{m, k}^{j}\right]$ and $\left[\tilde{\theta}_{m, k}^{j}\right]$ to evaluate 26,27

Lemma 3 can decouple the unknown matrix $\left[\theta_{m, k}^{j}\right]$ with a large size. In this case, we only need to calculate the cumulative average $\mathrm{EH}$ rate of the $k$-th parent relay once, instead of calculating it for each child relay (i.e. the $(m, k)$ th child relay for all $m$ ) in FP5. Particularly, the cumulative average EH rate of the $k$-th relay is $\bar{P}_{k, j}^{\mathrm{EH}}=\frac{\sum_{i=1}^{j} \Psi_{k, i}}{j}$.

With the help of the two lemmas, we can obtain a new problem whose feasibility is equivalent to FP5, while is much easier to check. The results are in the following Corollary 1 . which can be directly proved utilizing Theorem 1 and Lemmas 2 and 3

Corollary 1. The feasibility of FP5 is equivalent to the feasibility of the following problem for all $j \in \mathcal{J}$ :

FP6:

$$
\begin{array}{ll}
\text { find } & \phi_{m, k}, \tilde{\theta}_{m, k}^{j}, \forall k \in \mathcal{K}, m \in \mathcal{M} \\
\text { s.t. } & \sum_{k \in \mathcal{S}_{\eta, m}} \frac{2 \tilde{\theta}_{m, k}^{j} \bar{P}_{k, j}^{\mathrm{EH}} j N^{\mathrm{C}}+\frac{2 \phi_{m, k} E_{k}^{\mathrm{init}}}{T^{\mathrm{C}}}-2 \hat{P}_{m, k}(\eta)}{j N^{\mathrm{C}} \hat{P}_{m, k}(\eta)} \geq 1, \\
& \forall m \in \mathcal{M}, \\
& 0 \leq \phi_{m, k} \leq 1,0 \leq \tilde{\theta}_{m, k}^{j} \leq 1, \forall m \in \mathcal{M}, k \in \mathcal{K}, \\
& \sum_{m=1}^{M} \phi_{m, k}=1, \sum_{m=1}^{M} \tilde{\theta}_{m, k}^{j}=1, \forall k \in \mathcal{K} .
\end{array}
$$

Remark 3. We have obtained a highly simplified version of the feasibility problem from the challenging original one while most steps are equivalent, except for the approximation accompanied by employing Theorem 1. For FP6, it is a linear programming problem, with the complexity linear with $\sqrt{N^{E}} M K$. Moreover, the variable size is greatly reduced. We just need to solve FP6 for all $j \in \mathcal{J}$ separately, and check if FP6 is feasible for all $j \in \mathcal{J}$.

\section{B. Proposed Power Assignment and Relay Selection Algorithm}

So far, the feasibility problem for the epigraph form of problem OP1 with a given $\eta$ is obtained. The following problem can provide a sub-optimal solution for OP1

$$
\begin{aligned}
\min _{\left[\phi_{m, k}\right],\left[\tilde{\theta}_{m, k}^{j}\right], \eta} & \\
\text { s.t. } & \text { Constraints } 30] \sim 32 .
\end{aligned}
$$

Specifically, if OP3 is feasible, then OP1 is feasible, and the optimal value of OP3 provides a performance lower bound to OP1. According to the two-phase pattern of the feasibility for OP3, we can use a bisection method to find its optimal solution, which provides a sub-optimal solution for OP1. The proposed algorithm is shown as Algorithm 1, where $\epsilon$ represents the required accuracy.

In Algorithm 1, we first obtain the upper bound and lower bound for bisection. The lower bound for the source transmit power $\eta$ is obtained by having all the relays transmit with $\overline{\text { Algorithm 1: } \text { Suboptimal power assignment and relay selec- }}$ tion for OP1.

\section{Initialization:}

Obtain $\eta_{m, k}^{\mathrm{L}}$ by solving $U_{t h}=U_{m, k}\left(\eta_{m, k}^{\mathrm{L}}, P_{k, \max }^{\mathrm{tr}}\right)$. If no feasible solution ${ }^{4}$, set $\eta_{m, k}^{\mathrm{L}}=0$.

Obtain $\eta_{m, k, j}^{\mathrm{U}}$ by solving $U_{t h}=U_{m, k}\left(1 / \eta_{m, k, j}^{\mathrm{U}}, \Psi_{k, j}\right)$. If no feasible solution ${ }^{4}$, set $\eta_{m, k, j}^{\mathrm{U}}=0$.

Set $\eta^{\mathrm{L}} \leftarrow \max _{k \in \mathcal{K}, m \in \mathcal{M}} \eta_{m, k}^{\mathrm{L}}, \tilde{\eta}^{\mathrm{U}} \leftarrow \min _{k \in \mathcal{K}} \max _{m \in \mathcal{M}, j \in \mathcal{J}} \eta_{m, k, j}^{\mathrm{U}}$, $\eta^{\mathrm{U}}=1 / \tilde{\eta}^{\mathrm{U}}$.

While $\eta^{\mathrm{U}}-\eta^{\mathrm{L}}>\epsilon$

$\eta^{\mathrm{M}} \leftarrow \frac{1}{2}\left(\eta^{\mathrm{U}}+\eta^{\mathrm{L}}\right)$

Obtain $\mathcal{S}_{\eta, m}, \hat{P}_{m, k}(\eta), \forall m \in \mathcal{M}, k \in \mathcal{K}$ by 22, and 24, respectively.

If problem FP6 is feasible for every $j \in \mathcal{J}$

$$
\begin{aligned}
\eta^{\mathrm{U}} & \leftarrow \eta^{\mathrm{M}} . \\
\eta^{\mathrm{L}} & \leftarrow \eta^{\mathrm{M}} .
\end{aligned}
$$

\section{End if}

\section{End while}

\section{Result:}

The desired sub-optimal $\eta$ is $\eta^{\mathrm{U}}$;

the transmit power matrix is $\mathbf{P}=\left[P_{m, k}^{\mathrm{tr}}\right]$ with $P_{m, k}^{\mathrm{tr}}=$ $\hat{P}_{m, k}(\eta)$;

the relay pre-selection is $\mathcal{S}_{m}=\mathcal{S}_{\eta, m}$;

in each transmission block, the $m$-th source selects one active relay (according to Eq. (3)) randomly from $\mathcal{S}_{m}$.

their peak power, while the upper bound is obtained by having an arbitrary relay transmit with its EH rate. Next, during each loop of the bisection, with a given $\eta$, we determine a temporary relay pre-selection result $\mathcal{S}_{\eta}$, and a temporary power assignment matrix $\hat{\mathbf{P}}_{\eta}=\hat{P}_{m, k}(\eta)$. Then, the feasibility of FP6 is checked for all $j \in \mathcal{J}$. If it is feasible, we reduce the value of $\eta$; otherwise, we increase it. The smallest feasible $\eta$ is the sub-optimal value of OP1. Note that in the result of Algorithm 11, relay pre-selection and power assignment are fixed within the whole transmission duration, while relay selection needs to be performed at the beginning of each transmission block.

Remark 4. The proposed cooperation strategy with joint power assignment and relay selection has a low computational complexity, and also a low requirement on the side information. Particularly, only the statistical channel side information is needed. Furthermore, when the relay number is very large, the offline EH information can also be exempted. In contrast, the previously proposed transmission strategies for EH networks are not quite practical, such as the offline approach that requires all the offline side information [11], [12- -14], [29], or the online algorithm with a high computational complexity [16. 18]. Moreover, as will be shown in the simulation, the performance of Algorithm [1] is close to optimal. On the other hand, this approach is very general in the sense that

\footnotetext{
${ }^{4}$ If the equation has no feasible solution, it means that some relays have too poor relay-destination channels. Consequently, even if the source uses an infinite transmit power, the required utility still cannot be satisfied.
} 
the development is independent of the actual utility function expression, as long as it is monotonic.

\section{Simulation Results}

In this section, we will provide simulation results to demonstrate the performance of the proposed cooperation strategy. As shown in Fig. 11 in the simulation we consider a rectangular area with length $L_{x}$ and width $L_{y}$, respectively. Without loss of generality, the bottom-left of the rectangle is set as the origin. The sources and destinations are located on two opposite sides, with the coordinates given as $\left(0, \frac{m}{M+1} L_{y}\right)$ for the $m$ th source, while $\left(L_{x}, \frac{m}{M+1} L_{y}\right)$ for the $m$-th destination. We assume that all the relays are uniformly distributed inside the rectangle.

In particular, we consider both the free-space path loss and small scale fading. Denote the reference distance for the path loss as $d_{0}=10 \mathrm{~m}$, and the free-space path loss at $d_{0}$ for a carrier frequency $2.4 \mathrm{GHz}$ is calculated as $60 \mathrm{~dB}$ based on $[\lambda /(4 \pi d)]^{2}$, where $\lambda$ is the wavelength. We set $L_{x}=$ $L_{y}=10 d_{0}$. The small scale fading is Rayleigh fading, with scale parameter 1 . The channel bandwidth is $B=1 \mathrm{MHz}$, and noise power spectral density is $N_{0}=10^{-16} \mathrm{~W} / \mathrm{Hz}$, while the transmission block length $T^{\mathrm{C}}$ is $10 \mathrm{~ms}$. Unless otherwise mentioned, we set the $\mathrm{EH}$ interval as $T^{\mathrm{E}}=5 T^{\mathrm{C}}$, and the total transmission duration as $T=5 T^{\mathrm{E}}$. For each realization, the EH rate of each relay is drawn randomly with uniform distribution in $\left[\bar{P}^{\mathrm{EH}}(1-\alpha), \bar{P}^{\mathrm{EH}}(1+\alpha)\right]$, where $\bar{P}^{\mathrm{EH}}$ is the average $\mathrm{EH}$ rate, and $0<\alpha<1$. In the simulation, the default value of $\alpha$ is 0.5 , while the maximum transmit power for each relay and the average source transmit power are set as $P_{k, \text { max }}^{\mathrm{tr}}=2 \mathrm{~W}$ and $\bar{P}^{\mathrm{EH}}=20 \mathrm{~mW}$, respectively.

\section{A. The Single S-D Pair Case}

To demonstrate the advantage of the proposed approach, we will compare it with a greedy policy, which will always select the relay that can provide the best performance within each transmission block, while the selected relay exhausts its energy. Such a policy does not take the full EH constraints into consideration. We will also compare with a performance upper bound, which, different from Algorithm 11. solves the linear programming (LP) relaxation of FP2 instead of using our proposed sufficient condition 20. In the simulation, we adopt the successful transmission probability $\mathbb{P}^{\mathrm{s}}=1-\mathbb{P}^{\mathrm{o}}$ as the utility function, where $\mathbb{P}^{\circ}$ is the outage probability, and the outage probability with a single AF relay can be calculated based on Eq. (3.65) in [42]. The SNR threshold is set as $\gamma=1$. The required successful transmission probability is set as 0.99 . The source transmit powers versus the relay number for different policies are plotted in Fig. 2 As a reference, the transmit power of the direct link transmission without the assistance of any relay is calculated as $0.995 \mathrm{~W}$. We have the following observations from the simulation results:

- As shown in Fig. 2, the transmit power of the proposed policy decreases with the relay number, and it is much smaller than what is needed with the direct link transmission, which is $0.995 \mathrm{~W}$. The performance of the proposed algorithm is close to that of the performance upper

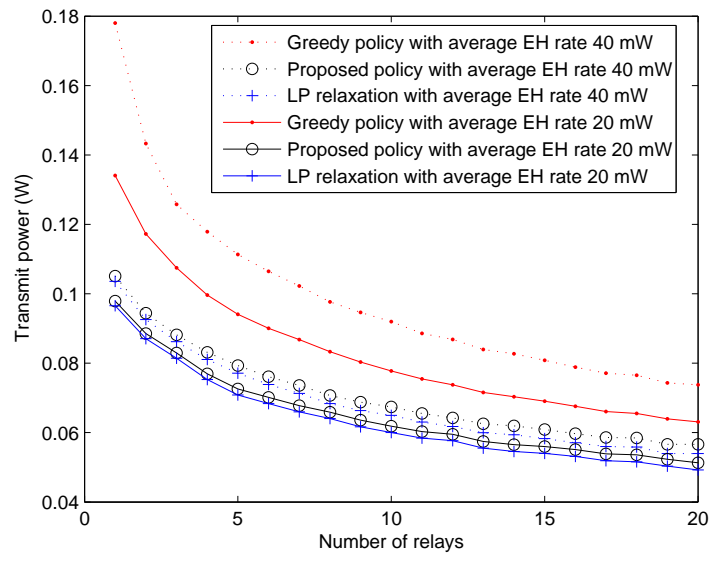

Figure 2: Source transmit power versus the relay number, with two average $\mathrm{EH}$ rates as $20 \mathrm{~mW}$ and $40 \mathrm{~mW}$, respectively.

bound, which reveals the effectiveness of the proposed methodology.

- The proposed policy outperforms the greedy policy which only considers the current transmission block. For example, with average $\mathrm{EH}$ rate $20 \mathrm{~mW}$, to have the source transmit power less than $0.08 \mathrm{~W}$, the proposed policy requires around 5 relays, while the best-effort policy needs around 15 relays.

- We see that increasing either the relay number or the average $\mathrm{EH}$ rate can reduce the required source transmit power. Meanwhile, increasing the relay number has a more obvious effect. The intuitive explanation is that doubling the relay number will not only improve the total available energy of all EH relays, but also reduce the (S-R or R-D) transmission distances on average for each pair.

In the following, we will check the two asymptotic properties in Theorem [1. i.e. the necessity property and the deterministic property.

For the necessity property, the source transmit power versus transmission block numbers with 5 relays are shown in Fig. 3. We can see that even when there are only 10 transmission blocks in each $\mathrm{EH}$ interval, the gap with the performance upper bound is already small. When there are more than 100 transmission blocks, the difference becomes indistinguishable from the figure. As in practical systems, for the EH resources such as solar energy [18, 35], the EH interval (many seconds to even several minutes) are normally much larger than the transmission block length (milliseconds to several hundred milliseconds). In such cases, the performance of our policy will be quite close to optimal. Note that, with the increase of transmission blocks, the performances of all curves degrades gradually, which is due to our fixed initial energy value setting, and a larger number of transmission blocks means less available energy on average.

Next, we check the deterministic property. Without the offline energy side information, due to the randomness of future energy arrivals, inevitably we can no longer deterministically guarantee all the constraints are satisfied. In general, some performance loss will happen. In particular, since we 


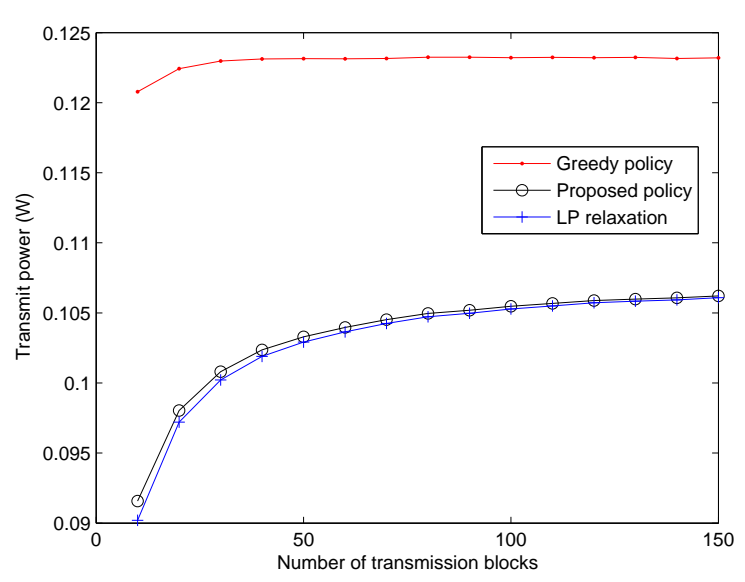

Figure 3: Source transmit power versus the number of transmission blocks with 5 relays.

decide the transmit power/relay pre-selection based only on the current side information, it is possible that we under-estimate or over-estimate the future relay energy harvesting condition. Naturally, we may set a too high or too low source transmit power, and this will possibly then bring about higher source power consumption, or energy outage, respectively. In the following, these two kinds of performance loss will be discussed separately, and shown to become negligible when increasing the relay number. In the case of under-estimation of future relay $\mathrm{EH}$ situation, from the second $\mathrm{EH}$ interval, there will be some energy waste for the source. The average transmit power for this under-estimation case 5 with $N^{\mathrm{C}}=1000$ is shown in Fig. (4). We can see that when the relay number is larger than 6, the gap is already very small. For the case of over-estimation of future relay EH situation, we may assign the source a too low transmit power, and thus in some future EH intervals, the required QoS cannot be guaranteed deterministically, which is called the energy outage. In the simulation, we count the respective ratio of transmission blocks not suffering energy outage. The average ratio of no energy outage for the policy using only current EH rates with $N^{\mathrm{C}}=1000$ is shown in Fig. 4 (b). We can see that this ratio is no smaller than 0.985 , and is approaching 1 with the increase of the relay number.

\section{B. The Multiple S-D Pair Case}

For the general case with multiple S-D pairs, we will also make a comparison with a greedy policy, for which, in each transmission block, relay selection is executed to minimize the instantaneous source transmit powers sequentially for all the S-D pairs in the current transmission block. On the other hand, we compare both policies with a performance upper bound, for which the feasibility is checked by solving the LP relaxation of OP1 instead of solving OP1 with Algorithm 11 The source transmit powers for different policies versus the number of relays with 3 S-D pairs, and versus the number of S-D pairs with 5 relays are plotted in the Fig. 5. We have the following observations from the simulation results:

\footnotetext{
${ }^{5}$ When calculating the average transmit power in the following simulation, we only take into account the realizations where we have under-estimated the future energy arrival and assign a too high power to the source.
}

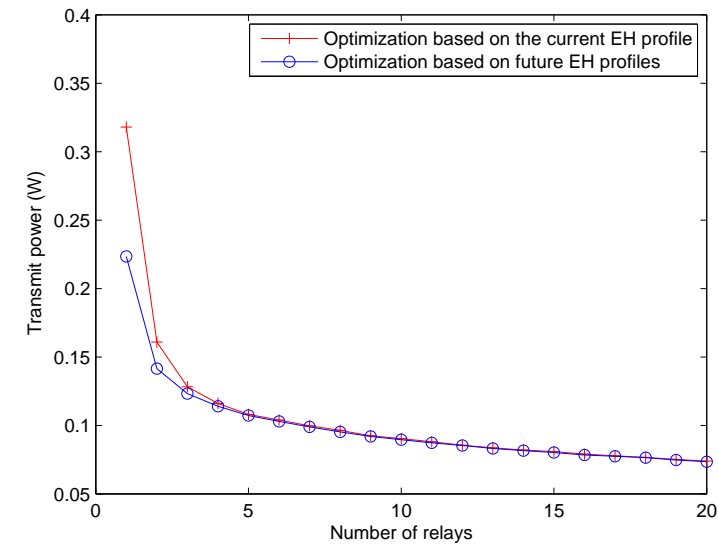

(a) The average transmit power for the under-estimation case versus the number of relays.

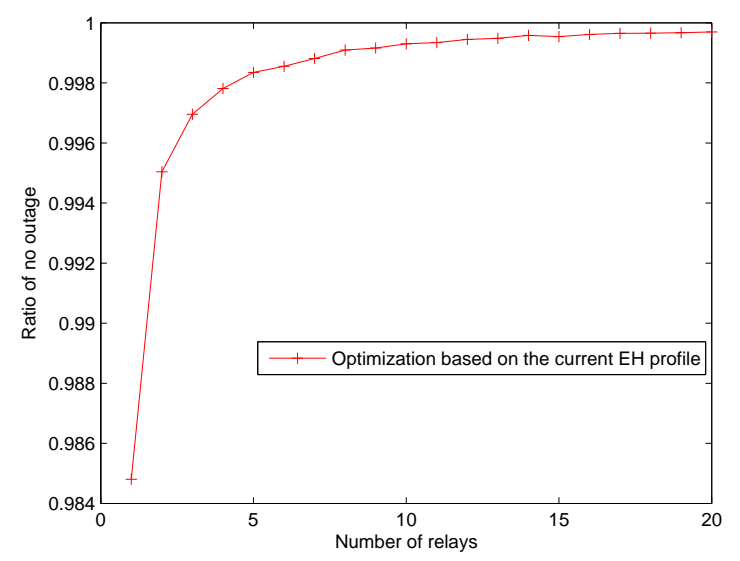

(b) The average ratio of no energy outage for the policy using only current EH rates versus the number of relays.

Figure 4: Performance loss of using only the current EH profiles.

- As shown in Fig. 5 (a), for the multi-pair system, the transmit powers also decrease with the number of relays, and to have the same source transmit power more relays will be needed than the single-pair case. The performance of the proposed policy is quite close to that of the LP relaxation, which demonstrates the effectiveness of Algorithm 1

- The greedy policy has a performance loss compared to the proposed policy, as it only takes the current transmission block into consideration. For example, to have the source transmit power less than $0.1 \mathrm{~W}$, the proposed policy requires more than 6 relays, while the greedy policy requires more than 13 relays.

- From Fig. 5 (b), as the number of S-D pairs increases, both the proposed policy and the greedy policy will experience performance degradation, as on average the number of relays that can assist each pair decreases. Meanwhile, the gap between the two policies increases with the number of S-D pairs. Thus, the proposed policy is more advantageous with a large number of S-D pairs. 


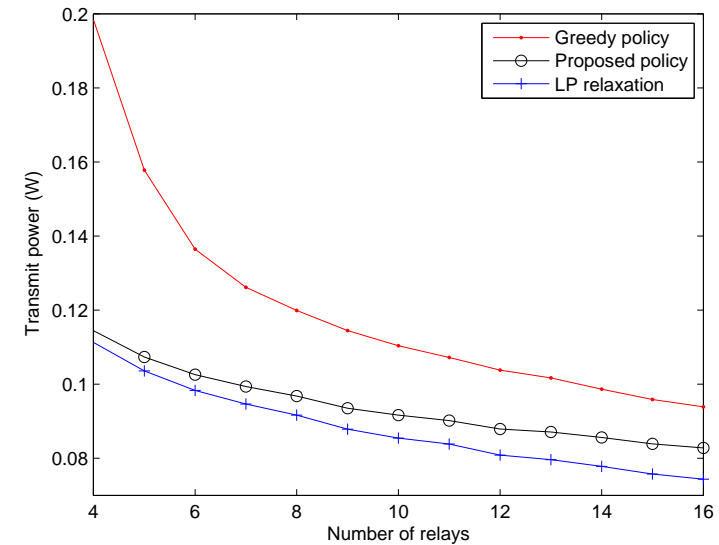

(a) Source transmit power versus the number of relays.

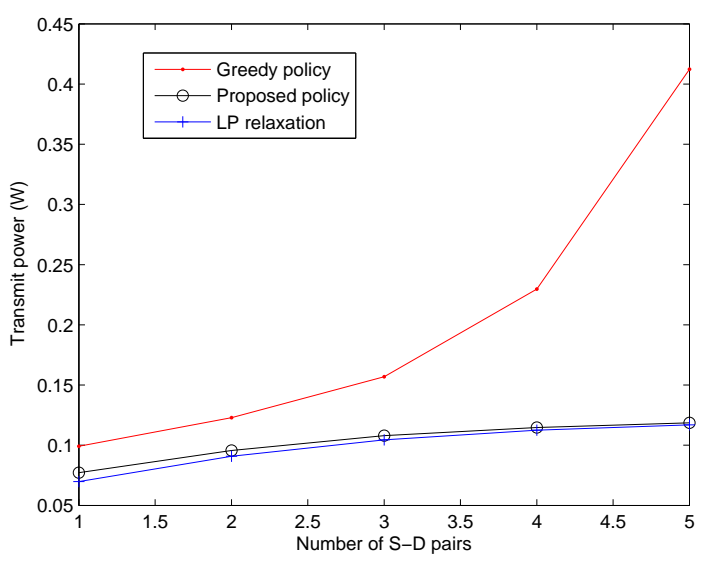

(b) Source transmit power versus the number of S-D pairs.

Figure 5: Source transmit power for the multi-pair case.

\section{CONClusions AND Future WORKS}

In this work, we proposed to achieve energy savings for a conventional wireless network by providing energy diversity via multiple EH relays. The proposed cooperation strategy is of low complexity and only depends on the statistical channel side information. Particularly, an energy hardening effect was revealed, which has demonstrated that it is possible to relax the requirement of the offline $\mathrm{EH}$ information, and this effect hence greatly simplifies the system design. Simulation results have demonstrated that such a simple cooperation strategy can overcome the low $\mathrm{EH}$ rate at each single EH relay, and provide significant power gain to improve the sourcedestination communication. For the future work, it would be interesting to apply the proposed algorithms to other $\mathrm{EH}$ networks.

\section{APPENDIX PROOF FOR THEOREM 1}

The proof is divided into three parts: The sufficiency of Eq. (20), the necessity property, and the deterministic property.

1. Sufficiency of Eq. (20).

To show the feasibility of FP3, we need to show that with 20. satisfied, we can always find a feasible solution $\mathrm{Z}=\left[z_{k, n}\right]$ such that all the constraints of $\mathbf{F P 3}$ are satisfied. All the relays are active for the first transmission block due to the initial energy setting. Thus, we only need to prove the existence of a feasible relay selection result for the $(L+1)$-th transmission block, with $1 \leq L \leq N-1$. Moreover, the $L$-th transmission block is in the $l$-th $\mathrm{EH}$ interval, with $l=\left\lceil L / N^{C}\right\rceil$. For convenience, denote the number of transmission blocks when the $k$-th relay is selected during the first $L$ transmission blocks as $n_{k, L}$. For the first $L$ transmission blocks, we have $\sum_{k \in \mathcal{S}_{\eta}} n_{k, L} \leq L$, where the equality is achieved if there exist active relays (i.e. satisfying Eq. (3) ) for all these $L$ transmission blocks. Furthermore, based on (20), $\forall l$ such that $1 \leq l \leq N^{\mathrm{E}}$, we can verify

$$
\sum_{k \in \mathcal{S}_{\eta}}\left(\frac{\sum_{i=1}^{l} \Psi_{k, i}}{\hat{P}_{k}(\eta)}+\frac{E_{k}^{\mathrm{init}} / T^{\mathrm{C}}-\hat{P}_{k}(\eta) / 2}{\hat{P}_{k}(\eta) N^{\mathrm{C}}}\right) \geq \frac{l}{2},
$$

and

$$
\sum_{k \in \mathcal{S}_{\eta}}\left(\frac{\sum_{i=1}^{l+1} \Psi_{k, i}}{\hat{P}_{k}(\eta)}+\frac{E_{k}^{\mathrm{init}} / T^{\mathrm{C}}-\hat{P}_{k}(\eta) / 2}{\hat{P}_{k}(\eta) N^{\mathrm{C}}}\right) \geq \frac{l+1}{2} .
$$

Now, denote $x=\frac{L-l N^{\mathrm{C}}}{N^{\mathrm{C}}}$, and $y=1-x$, then by calculating $x$ Eq. 333 $+y$ Eq. 34, and adopting Eq. $\quad 1$, we have $\sum_{k \in \mathcal{S}_{\eta}}\left(2 \sum_{n=1}^{L} \frac{P_{k, n}^{\mathrm{EH}}}{\hat{P}_{k}(\eta)}\right.$ $\left.+2 \frac{E_{k}^{\text {init }} / T^{\mathrm{C}}-\hat{P}_{k}(\eta) / 2}{\hat{P}_{k}(\eta)}\right) \geq L \geq \sum_{k \in \mathcal{S}_{\eta}} n_{k, L}$. That is, $\sum_{k \in \mathcal{S}_{\eta}}\left(2 \frac{\sum_{n=1}^{L} P_{k, n}^{\mathrm{EH}}+E_{k}^{\mathrm{init}} / T^{\mathrm{C}}-\hat{P}_{k}(\eta) / 2}{\hat{P}_{k}(\eta)}-n_{k, L}\right)^{2} \geq 0$. Following the pigeonhole principle, we can verify that there exists at least one $\hat{k} \in \mathcal{S}_{\eta}$, such that $2 \frac{\sum_{n=1}^{L} P_{\hat{k}, n}^{\mathrm{EH}}+E_{\hat{k}}^{\text {init }} / T^{\mathrm{C}}-\hat{P}_{\hat{k}}(\eta) / 2}{\hat{P}_{\hat{k}}(\eta)}-n_{\hat{k}, L} \geq 0, \quad$ i.e. $E_{\hat{k}}^{\mathrm{init}}+\sum_{n=1}^{L^{k}} P_{\hat{k}, n}^{\mathrm{EH}} T^{\mathrm{C}}-\hat{P}_{\hat{k}}(\eta) n_{\hat{k}, L} \frac{T^{\mathrm{C}}}{2} \geq \hat{P}_{\hat{k}}(\eta) \frac{T^{\mathrm{C}}}{2}$. This means that the remaining energy of the $\hat{k}$-th relay after $L$ transmission blocks is enough to support its assigned transmit power. That is, it is active based on Eq. (3) and ready for the relay selection of the $(L+1)$-th transmission block. We can then set $z_{\hat{k}, L+1}=1$ while $z_{k, L+1}=0$ for $k \neq \hat{k}, k \in \mathcal{K}$, for the $(L+1)$-th transmission block. It can be verified that the obtained relay selection result for $L \in \mathcal{N}$ satisfies all the constraints. Hence, this is a feasible solution for FP3.

\section{Necessity property.}

It can be verified that the following inequality is a sufficient condition for Eq. 20): $\zeta_{1}(\eta, l)=\sum_{k \in \mathcal{S}_{\eta}} \frac{2 \bar{\Psi}_{k, l}}{\hat{P}_{k}(\eta)} \geq 1, \forall l$. Therefore, we only provide the necessity of this inequality, instead of Eq. 20, when $N^{\mathrm{C}} \rightarrow \infty$. We will do so by contradiction. Specifically, assume that there exists one EH interval, e.g., the $l^{*}$-th, say,

$$
\sum_{k \in \mathcal{S}_{\eta}} \frac{2 \bar{\Psi}_{k, l^{*}}}{\hat{P}_{k}(\eta)}<1
$$

but the feasibility of FP3 still holds. Similar to the proof for the sufficiency property, we denote the number of blocks that the $k$-th relay is selected during the first $L=l^{*} T^{\mathrm{c}}$ 
transmission blocks as $n_{k, L}$. Due to Eq. (17) of FP3, there exist active relays for all the $L$ transmission blocks. Thus,

$$
\sum_{k \in \mathcal{S}_{\eta}} n_{k, L}=L .
$$

By jointly considering (35) and 36, we have $\sum_{k \in \mathcal{S}_{\eta}} \frac{2 \bar{\Psi}_{k, l^{*}}}{\hat{P}_{k}(\eta)} L<L=\sum_{k \in \mathcal{S}_{\eta}} n_{k, L}$, i.e. $\sum_{k \in \mathcal{S}_{\eta}}\left(\frac{2 \bar{\Psi}_{k, l^{*}} L}{\hat{P}_{k}(\eta)}\right.$ $\left.-n_{k, L}\right)<0$. Following the pigeonhole principle, we can verify that there exists at least one $\hat{k} \in \mathcal{S}_{\eta}$, such that $\frac{2 \bar{\Psi}_{\hat{k}, l l} L}{\hat{P}_{\hat{k}}(\eta)}-n_{\hat{k}, L}<0$, with $L=l^{*} N^{\mathrm{C}} \rightarrow \infty, \frac{E_{\hat{k}}^{\mathrm{init}^{k}}+2 \bar{\Psi}_{\hat{k}, l^{*}} L}{\hat{P}_{\hat{k}}(\eta)}-n_{\hat{k}, L}<0$, i.e. $E_{\hat{k}}^{\text {init }}+2 \bar{\Psi}_{\hat{k}, l^{*}} L-n_{\hat{k}, L} \hat{P}_{\hat{k}}(\eta)<0$. This violates the energy causality constraint (5). Therefore, the feasibility of FP3 is violated.

\section{Deterministic property.}

Denote $X_{k, l}=\mathbf{1}_{U_{k}\left(\eta, P_{k, \text { max }}^{\mathrm{tr}}\right) \geq U_{\mathrm{th}}} \frac{2 \bar{\Psi}_{k, l} l N^{\mathrm{C}}+2 E_{k}^{\mathrm{init}} / T^{\mathrm{C}}-2 \hat{P}_{k}(\eta)}{l N^{\mathrm{C}}}$

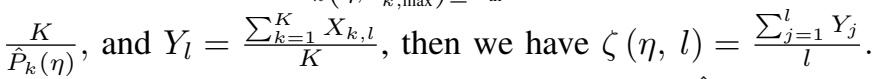
With a given $l$, and given locations, (i.e. given $\hat{P}_{k}(\eta)$ ), $X_{k, l}$ with different $k$ are independent of each other, as all the EH rates for different relays are independent. We can further verify that all $X_{k, l}$ are with finite variances, as $\sigma_{X_{k, l}}^{2} \leq\left(E\left[X_{k, l}\right]\right)^{2} \leq$ $\left(\frac{2 E\left[\bar{\Psi}_{k, l}\right] l N^{\mathrm{C}}+2 E_{k}^{\mathrm{init}} / T^{\mathrm{C}}-2 \hat{P}_{k}(\eta)}{l N^{\mathrm{C}}} \frac{K}{\hat{P}_{k}(\eta)}\right)^{2} \leq\left(\operatorname{constant} \frac{K}{\hat{P}_{k}(\eta)}\right)^{2}$, while the finite value of $\frac{K}{\hat{P}_{k}(\eta)}$ can be verified non-trivially by the two-phase pattern for the feasibility of FP1, as mentioned in the end of the last section. Then, $\forall l, \sum_{k} \sigma_{X_{k, l}}^{2} / k^{2}<\infty$. Based on the strong law of large numbers, cf. Theorem 3 in Chapter 2.9 in [43], $Y_{j}$ converges to be a deterministic value. It can be further verified that $\zeta(\eta, l)=\frac{\sum_{j=1}^{l} Y_{j}}{l}$ will become deterministic.

\section{REFERENCES}

[1] Z. Hasan, H. Boostanimehr, and V. K. Bhargava, "Green cellular networks: A survey, some research issues and challenges," IEEE Commun. Surveys Tutorials, vol. 13, no. 4, pp. 524-540, 2011.

[2] S. Ulukus, A. Yener, E. Erkip, O. Simeone, M. Zorzi, P. Grover, and K. Huang, "Energy harvesting wireless communications: A review of recent advances," IEEE J. Sel. Areas Commun., vol. 33, no. 3, pp. 360381, 2015.

[3] Y. Mao, Y. Luo, J. Zhang, and K. B. Letaief, "Energy harvesting small cell networks: feasibility, deployment, and operation," IEEE Commun. Mag., vol. 53, no. 6, pp. 94-101, Jun. 2015.

[4] J. Paradiso and T. Starner, "Energy scavenging for mobile and wireless electronics," IEEE Pervasive Comput., vol. 4, no. 1, pp. 18-27, Jan.-Mar. 2005.

[5] K. Lin, J. Yu, J. Hsu, S. Zahedi, D. Lee, J. Friedman, A. Kansal, V. Raghunathan, and M. Srivastava, "Heliomote: Enabling long-lived sensor networks through solar energy harvesting," in Proc. ACM SenSys, San Diego, CA, 2005.

[6] X. Jiang, J. Polastre, and D. Culler, "Perpetual environmentally powered sensor networks," in Proc. IPSN, Los Angeles, CA, Apr. 2005, pp. $463-$ 468.

[7] S. Sudevalayam and P. Kulkarni, "Energy harvesting sensor nodes: survey and implications," IEEE Commun. Surveys Tuts., vol. 13, no. 3, pp. 443-461, 2011.

[8] Q. Zhang, A. Agbossou, Z. Feng, and M. Cosnier, "Solar microenergy harvesting based on thermoelectric and latent heat effects part II: Experimental analysis," Sensors and Actuators A: Physical, vol. 163, no. 1, pp. 284-290, Sept. 2010.
[9] K. T. Phan, T. Le-Ngoc, S. A. Vorobyov, and C. Tellambura, "Power allocation in wireless multi-user relay networks," IEEE Trans. Wireless Comm., vol. 8, no. 5, pp. 2535-2545, May 2009.

[10] T. Himsoon, W. P. Siriwongpairat, Z. Han, and K. J. R. Liu, "Lifetime maximization via cooperative nodes and relay deployment in wireless networks," IEEE J. Sel. Areas Commun., vol. 25, no. 2, pp. 306-317, Feb. 2007.

[11] J. Yang and S. Ulukus, "Optimal packet scheduling in an energy harvesting communication system," IEEE Trans. Commun., vol. 60 , no. 1, pp. 220-230, Jan. 2012.

[12] M. A. Antepli, E. U. Biyikoglu, and H. Erkal, "Optimal packet scheduling on an energy harvesting broadcast link," IEEE J. Sel. Areas Commun., vol. 29, no. 8, pp. 1721-1731, Sept. 2011.

[13] J. Yang and S. Ulukus, "Optimal packet scheduling in a multiple access channel with rechargeable nodes," in Proc. IEEE Int. Conf. Commun., Kyoto, Japan, Jun. 2011.

[14] K. Tutuncuoglu and A. Yener, "Sum-rate optimal power policies for energy harvesting transmitters in an interference channel," JCN Special Issue on Energy Harvesting in Wireless Networks, vol. 14, no. 2, pp. 151-161, Apr. 2012.

[15] Y. Luo, J. Zhang, and K. B. Letaief, "Training optimization for energy harvesting communication systems," in Proc. IEEE Globecom, Anaheim, CA, Dec. 2012.

[16] C. K. Ho and R. Zhang, "Optimal energy allocation for wireless communications with energy harvesting constraints," IEEE Trans. Signal Process., vol. 60, no. 9, pp. 4808-4818, Sep. 2012.

[17] O. Ozel, K. Tutuncuoglu, J. Yang, S. Ulukus, and A. Yener, "Transmission with energy harvesting nodes in fading wireless channels: Optimal policies," IEEE J. Sel. Areas Commun., vol. 29, no. 8, pp. 1732-1743, Sept. 2011.

[18] C. Huang, R. Zhang, and S. Cui, "Optimal power allocation for outage probability minimization in fading channels with energy harvesting constraints," IEEE Trans. Wireless Comm., vol. 13, no. 2, pp. 10741087, Feb. 2014.

[19] Y. Mao, J. Zhang, and K. B. Letaief, "A lyapunov optimization approach for green cellular networks with hybrid energy supplies," IEEE J. Select. Areas Commun., vol. 33, no. 12, pp. 2463-2477, Dec. 2015.

[20] E. Sendonaris, A.and Erkip and B. Aazhang, "User cooperation diversity-part I: System description and part II: Implementation aspects and performance analysis," IEEE Trans. Commun., vol. 51, no. 11, pp. 1927-1948, Nov. 2003.

[21] J. Laneman, D. Tse, and G. Wornell, "Cooperative diversity in wireless networks: Efficient protocols and outage behavior," IEEE Trans. Inf. Theory, vol. 50, no. 12, pp. 3062-3080, Dec. 2004.

[22] W. Chen, L. Dai, K. B. Letaief, and Z. Cao, "A unified cross-layer framework for resource allocation in cooperative networks," IEEE Trans. Wireless Comm., vol. 7, no. 8, pp. 3000-3012, Aug. 2008.

[23] K. B. Letaief and W. Zhang, "Cooperative communications for cognitive radio networks," Proceedings of the IEEE, vol. 97, no. 5, pp. 878-893, May 2009.

[24] X. Zhou, B. Bai, and W. Chen, "A low complexity energy efficiency maximization method for multiuser amplify-and-forward MIMO relay systems with a holistic power model," IEEE Communications Letters, vol. 18, no. 8, pp. 1371-1374, Jun. 2014.

[25] D. Gunduz and B. Devillers, "Two-hop communication with energy harvesting," in Proc. 4th International Workshop on Computational Advances in Multi-Sensor Adaptive Processing (CAMSAP), San Juan, PR, Dec. 2011.

[26] C. Huang, R. Zhang, and S. Cui, "Throughput maximization for the Gaussian relay channel with energy harvesting constraints," IEEE J. Sel. Areas Commun., vol. 31, no. 8, pp. 1469-1479, Aug. 2013.

[27] O. Orhan and E. Erkip, "Optimal transmission policies for energy harvesting two-hop networks," in Proc. 2012 Conf. Inform. Sciences and Systems, Princeton, NJ, Mar. 2012.

[28] Y. Luo, J. Zhang, and K. B. Letaief, "Optimal scheduling and power allocation for two-hop energy harvesting communication systems," IEEE Trans. Wireless Comm., vol. 12, no. 9, pp. 4729-4741, Sept. 2013.

[29] O. Orhan and E. Erkip, "Throughput maximization for energy harvesting two-hop networks," in Proc. IEEE International Symposium on Information Theory (ISIT), Istanbul, Turkey, Jul. 2013, pp. 1596-1600.

[30] I. Ahmed, A. Ikhlef, R. Schober, and R. K. Mallik, "Power allocation for conventional and buffer-aided link adaptive relaying systems with energy harvesting nodes," IEEE Trans. Wireless Commun., vol. 13, no. 3, pp. 1182-1195, Mar. 2014.

[31] Y. Mao, J. Zhang, and K. B. Letaief, "Joint link selection and relay power allocation for energy harvesting relaying systems," in Proc. Globecom, Austin, TX, Dec. 2014, pp. 2568-2573. 
[32] B. Medepally and N. B. Mehta, "Voluntary energy harvesting relays and selection in cooperative wireless networks," IEEE Trans. Wireless Commun., vol. 9, no. 11, pp. 3543-3553, Nov. 2010.

[33] I. Ahmed, A. Ikhlef, R. Schober, and R. K. Mallik, "Joint power allocation and relay selection in energy harvesting AF relay systems," IEEE Wireless Commun. Lett., vol. 2, no. 2, pp. 239-242, Apr. 2013.

[34] Y. Luo, J. Zhang, and K. B. Letaief, "Relay selection for energy harvesting cooperative communication systems," in Proc. Globecom, Atlanta, GA, Dec. 2013.

[35] M. Gorlatova, A. Wallwater, and G. Zussman, "Networking low-power energy harvesting devices: Measurements and algorithms," in Proc. IEEE INFOCOM, Shanghai, China, Apr. 2011.

[36] D. Tse and P. Viswanath, Fundamentals of Wireless Communication. Cambridge University Press, 2005.

[37] Y. Luo, J. Zhang, and K. B. Letaief, "Achieving energy diversity with multiple energy harvesting relays," in Int. Conf. on Wireless Commun. and Signal Processing (WCSP), Hefei, China,, Oct. 2014.

[38] W.-H. Kuo and W. Liao, "Utility-based resource allocation in wireless networks," IEEE Trans. Wireless Commun., vol. 6, no. 10, pp. 36003606, Oct. 2007.

[39] J. Lee and S. Leyffer, Eds., Mixed Integer Nonlinear Programming, ser. The IMA Volumes in Mathematics and its Applications (Book 154). Springer, 2012.

[40] D. W. Pentico, "Assignment problems: A golden anniversary survey," European Journal of Operational Research, vol. 176, no. 2, pp. 774 793, Jan. 2007.

[41] B. M. Hochwald, T. L. Marzetta, and V. Tarokh, "Multiple-antenna channel hardening and its implications for rate feedback and scheduling," IEEE Trans. Inf. Theory, vol. 50, no. 9, pp. 1893-1909, Sept. 2004.

[42] Y.-W. P. Hong, W.-J. Huang, and C.-C. J. Kuo, Cooperative Communications and Networking: Technologies and System Design. Springer, 2010.

[43] J. W. Lamperti, Probability: A survey of the mathematical theory, 2nd ed., ser. Wiley Series in Probability and Mathematical Statistics. Wiley, Aug. 1996.

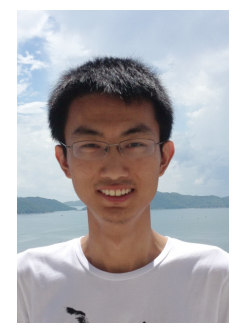

Yaming Luo (S'11) received his B.Eng. degree in the Department of Communication Engineering from the Harbin Institute of Technology, Harbin, China, in 2010. He also obtained the honor of Outstanding Graduates of Heilongjiang Province. He is currently working towards the Ph.D. degree in the Department of Electronic and Computer Engineering at the Hong Kong University of Science and Technology, under the supervision of Prof. Khaled B. Letaief. His current research interests include energy harvesting networks, relay systems, and green

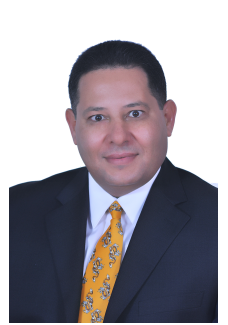

Khaled B. Letaief (S'85-M'86-SM'97-F'03) received the BS degree with distinction in Electrical Engineering (1984) from Purdue University, USA. $\mathrm{He}$ has also received the MS and Ph.D. Degrees in Electrical Engineering from Purdue University in 1986 and 1990, respectively.

From 1990 to 1993 , he was a faculty member at the University of Melbourne, Australia. He has been with the Hong Kong University of Science and Technology. While at HKUST, he has held numerous administrative positions, including the Head of the Electronic and Computer Engineering department, Director of the Center for Wireless IC Design, Director of Huawei Innovation Laboratory, and Director of the Hong Kong Telecom Institute of Information Technology. He has also served as Chair Professor and Dean of HKUST School of Engineering. Under his leadership, the School of Engineering has dazzled in international rankings (ranked \#14 in the world in 2015 according to QS World University Rankings).

From September 2015, he joined HBKU as Provost to help establish a research-intensive university in Qatar in partnership with strategic partners that include Northwestern University, Carnegie Mellon University, Cornell, and Texas A\&M.

Dr. Letaief is a world-renowned leader in wireless communications and networks. In these areas, he has over 500 journal and conference papers and given invited keynote talks as well as courses all over the world. He has made 6 major contributions to IEEE Standards along with 13 patents including 11 US patents.

He served as consultants for different organizations and is the founding Editor-in-Chief of the prestigious IEEE Transactions on Wireless Communications. He has served on the editorial board of other prestigious journals including the IEEE Journal on Selected Areas in Communications - Wireless Series (as Editor-in-Chief). He has been involved in organizing a number of major international conferences.

Professor Letaief has been a dedicated educator committed to excellence in teaching and scholarship. He received the Mangoon Teaching Award from Purdue University in 1990; HKUST Engineering Teaching Excellence Award (4 times); and the Michael Gale Medal for Distinguished Teaching (Highest university-wide teaching award at HKUST).

$\mathrm{He}$ is also the recipient of many other distinguished awards including 2007 IEEE Joseph LoCicero Publications Exemplary Award; 2009 IEEE Marconi Prize Award in Wireless Communications; 2010 Purdue University Outstanding Electrical and Computer Engineer Award; 2011 IEEE Harold Sobol Award; 2011 IEEE Wireless Communications Technical Committee Recognition Award; and 11 IEEE Best Paper Awards.

He had the privilege to serve IEEE in many leadership positions including IEEE ComSoc Vice-President, IEEE ComSoc Director of Journals, and member of IEEE Publications Services and Products Board, IEEE ComSoc Board of Governors, IEEE TAB Periodicals Committee, and IEEE Fellow Committee.

Dr. Letaief is a Fellow of IEEE and a Fellow of HKIE. He is also recognized by Thomson Reuters as an ISI Highly Cited Researcher.

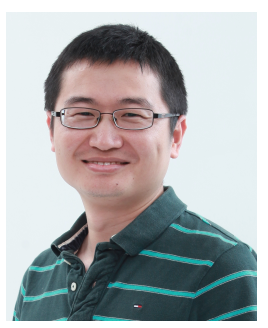

Jun Zhang (S'06-M'10-SM'15) received the B.Eng. degree in Electronic Engineering from the University of Science and Technology of China in 2004, the M.Phil. degree in Information Engineering from the Chinese University of Hong Kong in 2006, and the $\mathrm{Ph} . \mathrm{D}$. degree in Electrical and Computer Engineering from the University of Texas at Austin in 2009. He is currently a Research Assistant Professor in the Department of Electronic and Computer Engineering at the Hong Kong University of Science and Technology (HKUST). Dr. Zhang co-authored the book Fundamentals of LTE (Prentice-Hall, 2010). He received the 2014 Best Paper Award for the EURASIP Journal on Advances in Signal Processing, and the PIMRC 2014 Best Paper Award. He is an Editor of IEEE Transactions on Wireless Communications, and served as a MAC track co-chair for IEEE WCNC 2011. His research interests include wireless communications and networking, green communications, and signal processing. 